\title{
CP-RAYS IN SIMPLICIAL CONES
}

\author{
Leroy M. KELLY \\ Department of Mathematics, Michigan State University, East Lansing, MI 48824-1027, USA \\ Katta G. MURTY* \\ Department of Industrial and Operations Engineering, University of Michigan, Ann Arbor, \\ MI 48109-2117, USA
}

Layne T. WATSON**

Department of Computer Science, Virginia Polytechnic Institute and State University, Blacksburg, VA 24061, USA

Received 29 September 1988

Revised manuscript received 4 October 1989

This paper is dedicated to the memory of Paolo M. Camerini of Politecnico di Milano whose untimely passing is a great loss to his family and friends and to the optimization profession.

\begin{abstract}
An interior point of a triangle is called $C P$-point if its orthogonal projection on the line containing each side lies in the relative interior of that side. In classical mathematics, interest in the concept of regularity of a triangle is mainly centered on the property of every interior point of the triangle being a CP-point. We generalize the concept of regularity using this property, and extend this work to simplicial cones in $\mathbb{R}^{n}$, and derive necessary and sufficient conditions for this property to hold in them. These conditions highlight the geometric properties of $Z$-matrices. We show that these concepts have important ramifications in algorithmic studies of the linear complementarity problem. We relate our results to other well known properties of square matrices.
\end{abstract}

Key words: Simplicial cones, faces, orthogonal projections, CP-points and rays, linear complementarity problem, positive definite matrices, Z-matrices, P-matrices.

\section{Introduction}

Consider the equilateral triangle in $\mathbb{R}^{2}$ shown in Figure 1 . The point $b$ in Figure 1 satisfies the following properties.

(i) It is in the interior of the triangle.

(ii) For each side of the triangle, the orthogonal projection of $b$ on the straight line containing that side, is in the relative interior of that side.

We will call a point satisfying these two properties, a CP-point (abbreviation for "centrally (or interiorly) projecting point," since it projects into the interior of each side) for the triangle. See Figure 2 for a triangle in $\mathbb{R}^{2}$ and a point $c$ in it which is not a CP-point, since it violates (ii).

Every triangle in $\mathbb{R}^{2}$ has a CP-point. By a well-known result in classical geometry, the bisector lines of the three angles of a triangle have a common point, $b$, and clearly that point $b$ is a CP-point for the triangle. See Figure 2.

* Partially supported by NSF grants ECS-8521183 and ECS-8704052 and by NATO grant RG85-0240.

** Supported in part by Control Data Corporation grant 84V101 and AFOSR grant 85-0250. 


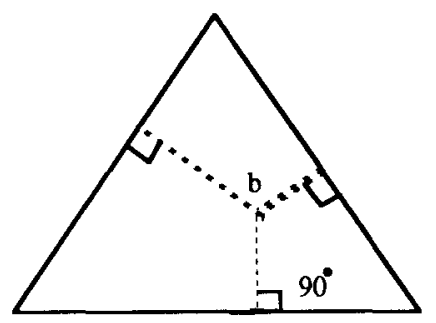

Fig. 1. An equilateral triangle and a CP-point $b$ in it.

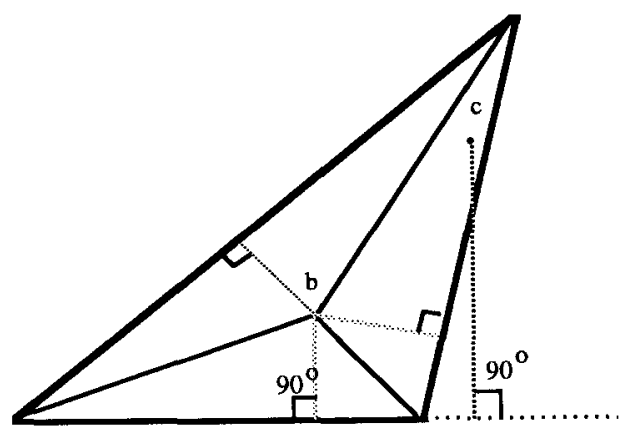

Fig. 2. A triangle in $\mathbb{R}^{2}$ and a point $c$ in it which is not a CP-point. However, the point $b$ where all the bisector lines of the angles meet, is a CP-point.

Also, it can be verified that every interior point of the equilateral triangle in Figure 1 is a CP-point for it, but this property does not hold for the triangle in Figure 2.

In this paper we generalize the concept of CP-points for triangles in $\mathbb{R}^{2}$ to simplicial cones in $\mathbb{R}^{n}$. We show that CP-points in simplicial cones play an important role in studies aimed at developing efficient algorithms for linear complementarity problems associated with positive definite symmetric matrices and P-matrices. We investigate several geometric properties associated with CP-points in simplicial cones, and relate them to classical results in matrix theory.

\section{Notation}

We will use the following notation.

LCP: Linear complementarity problem, defined in Section 4.

$\operatorname{LCP}(q, M)$ : An LCP for which the input data is the column vector $q$ and square matrix $M$.

$E_{i \bullet}, E_{\bullet j}:$ If $E$ is any matrix, $E_{i}$. denotes its $i$ th row vector, $E_{\circ j}$ denotes its $j$ th column vector.

$E_{\boldsymbol{L} J}:$ If $E=\left(e_{i j}\right)$ is any matrix of order $m \times n$, given $\boldsymbol{L} \subset\{1, \ldots, m\}, \boldsymbol{J} \subset\{1, \ldots, n\}$, $E_{\boldsymbol{L} J}$ denotes the submatrix $\left(e_{i j}: i \in \boldsymbol{L}, j \in \boldsymbol{J}\right)$ of $E$ determined by these subsets. 
$D_{J}$ : Submatrix of $D$ with row vectors $D_{i}$. for $i \in J$.

$D_{.}$: Submatrix of $D$ with column vectors $D_{. j}$ for $j \in \boldsymbol{J}$.

$x_{\boldsymbol{J}}$ : If $x=\left(x_{j}\right) \in \mathbb{R}^{n}, \boldsymbol{J} \subset\{1, \ldots, n\}, x_{\boldsymbol{J}}$ denotes the column vector $\left(x_{j}: j \in \boldsymbol{J}\right)$.

$\operatorname{Pos}(D)$ : Given the matrix $D$, this is the cone $\{x: x=D y$ for some $y \geqslant 0\}$.

LH $(D)$ : The linear hull of the set of column vectors of the matrix $D$, it is the subspace $\{x: x=D y$, for some vector $y\}$.

$I$ : Unit matrix of order $n$.

$|\boldsymbol{J}|$ : Cardinality of the set $\boldsymbol{J}$.

$\boldsymbol{K} \backslash \Delta$ : When $\boldsymbol{K}$ and $\Delta$ are two sets, $\boldsymbol{K} \backslash \Delta$ is the set of elements of $\boldsymbol{K}$ which are not in $\Delta$.

P-matrix: A square matrix, all of whose principal subdeterminants are $>0$.

Z-matrix: A square matrix, all of whose off-diagonal elements are $\leqslant 0$.

M-matrix: A Z-matrix which is also a P-matrix.

$P_{n}, Z_{n}, M_{n}$ : These are respectively the classes of $\mathrm{P}-, \mathrm{Z}$ - and M-matrices of order $n$.

$\|x\|:$ The Euclidean norm of the vector $x=\left(x_{j}\right)$, it is $+\sqrt{\sum_{j} x_{j}^{2}}$.

$\Gamma:$ The set $\{1, \ldots, n\}$.

Ray of $b$ : For $b \in \mathbb{R}^{n}, b \neq 0$, the ray of $b$ is $\operatorname{Pos}(b)=\{x: x=\alpha b$ for some $\alpha \geqslant 0\}$.

$\pi: \mathrm{Pi}$, the length of the circumference of a circle in $\mathbb{R}^{2}$ with diameter 1 .

PD: Positive definite.

\section{CP-Points and CP-rays in simplicial cones}

Let $D$ be a real square nonsingular matrix of order $n$. $\operatorname{Pos}(D)$ is a simplicial cone in $\mathbb{R}^{n}$. For each $j=1$ to $n$, the ray $\operatorname{Pos}\left(D_{\cdot j}\right)=\left\{x: x=\alpha D_{. j}, \alpha \geqslant 0\right\}$ is a generator or a generator ray for $\operatorname{Pos}(D)$. In this section we define CP-points and CP-rays for $\operatorname{Pos}(D)$, and various other geometric entities related to $\operatorname{Pos}(D)$, which are used later in studying CP-points.

Since $\operatorname{Pos}(D)$ is a simplicial cone, a face of $\operatorname{Pos}(D)$ is $\operatorname{Pos}(D . J)$ for some $J \subset \Gamma$ and vice versa. For any $\boldsymbol{J} \subset \Gamma$, we will say that $\operatorname{Pos}(D . J)$ is the face of $\operatorname{Pos}(D)$ corresponding to the subset $J$. When $|\boldsymbol{J}|=n-1, \operatorname{Pos}(D . J)$ is called a facet of $\operatorname{Pos}(D)$, it is a face of $\operatorname{Pos}(D)$ whose dimension is one less than the dimension of $\operatorname{Pos}(D)$.

The point $b \in \operatorname{Pos}(D)$ is said to be a CP-point for $\operatorname{Pos}(D)$ if it satisfies the following properties.

Property 1. $b$ is in the interior of $\operatorname{Pos}(D)$.

Property 2. For every face $\boldsymbol{F}$ of $\operatorname{Pos}(D)$, the orthogonal projection of $b$ on the linear hull of $\boldsymbol{F}$, is in the relative interior of $\boldsymbol{F}$.

Since there are $2^{n}-2$ nonempty proper faces of $\operatorname{Pos}(D)$, Property 2 consists of $2^{n}-2$ conditions. Here again, "CP-point" is an abbreviation for "centrally (or 
interiorly) projecting point," since this point projects into the relative interior of every face of $\operatorname{Pos}(D)$.

As an example, consider the simplicial cone, $\operatorname{Pos}(D)$ in $\mathbb{R}^{2}$, given in Figure 3, which is an obtuse angle. The point $\left(1, \frac{1}{4}\right)^{\mathrm{T}}$ violates Property 2 , since its orthogonal projection on the linear hull of $\operatorname{Pos}\left(D_{\cdot 1}\right)$ is $d$, which is not even in $\operatorname{Pos}\left(D_{\bullet_{1}}\right)$. The point $(0,1)^{\mathrm{T}}$ also violates Property 2 , since its orthogonal projection on the linear hull of $\operatorname{Pos}\left(D_{.2}\right)$ is 0 , which is not in the relative interior of $\operatorname{Pos}\left(D_{.2}\right)$. However, the point $b=(-1+\sqrt{2}, 1)^{\mathrm{T}}$ on the bisector line of the angle $\operatorname{Pos}(D)$ does satisfy both Properties 1 and 2, and is therefore a CP-point for $\operatorname{Pos}(D)$ in this example.

If $b$ is a CP-point for $\operatorname{Pos}(D)$, the ray $\operatorname{Pos}(b)=\{x: x=\alpha b$, for $\alpha \geqslant 0\}$ is said to be a $C P$-ray for $\operatorname{Pos}(D)$.

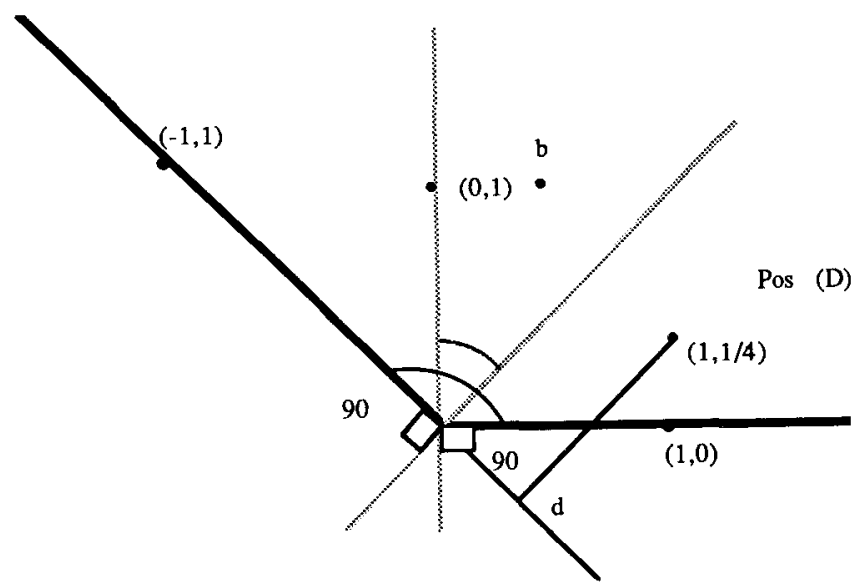

Fig. 3. $D=\left(\begin{array}{rr}-1 & 1 \\ 1 & 0\end{array}\right)$. Pos $(D)$ is the thick obtuse angled cone. The open cone bounded by the dashed lines, $\left\{y: D^{\mathrm{T}} y>0\right\}$ is the set of all CP-points for $\operatorname{Pos}(D)$.

Lemma 1. Every nonzero point on a CP-ray for $\operatorname{Pos}(D)$ is a CP-point for it.

Proof. By direct verification.

\subsection{Projection and non-projection faces of $\operatorname{Pos}(D)$ relative to a given point $b$}

Given $b \in \mathbb{R}^{n}$, the face $\operatorname{Pos}\left(D_{. J}\right)$ of $\operatorname{Pos}(D)$ corresponding to $J \subset \Gamma$, is said to be a projection face relative to $b$ if the orthogonal projection of $b$ in $\mathbf{L H}(D . J)$ is in the relative interior of $\operatorname{Pos}(D . J)$; non-projection face relative to $b$ otherwise.

The face of $\operatorname{Pos}(D)$ corresponding to $\Gamma$ is $\operatorname{Pos}(D)$ itself. By the above definition, $\operatorname{Pos}(D)$ is a projection face relative to $b$ iff $b$ is in the interior of $\operatorname{Pos}(D)$.

Thus $b$ is a CP-point iff every face of $\operatorname{Pos}(D)$ is a projection face relative to $b$, that is, iff there are no non-projection faces relative to $b$. 
The concept of a projection face is algorithmically very important in the study of the linear complementarity problem. In Murty and Fathi [16] and Wolfe [21] it has been used to develop efficient algorithms for nearest point problems in simplicial cones, and special types of linear complementarity problems. These algorithms, and consequently projection faces, play an important role in a new algorithm for linear programming developed by Chang and Murty [1].

\subsection{The set of CP-points of $\operatorname{Pos}(D)$ when $n=2$}

If $n=2$, and $\operatorname{Pos}(D)$ is an acute or right angle, every point in the interior of $\operatorname{Pos}(D)$ is a CP-point for it. If $\operatorname{Pos}(D)$ is an obtuse angle, any point $b$ in the interior of $\operatorname{Pos}\left(\left(D^{\mathrm{T}}\right)^{-1}\right)$ (these are points $b$ satisfying $D^{\mathrm{T}} b>0$ ) is a CP-point for $\operatorname{Pos}(D)$. See Figure 3.

In this case $(n=2)$, the bisector ray of the angle $\operatorname{Pos}(D)$, is always a CP-ray for $\operatorname{Pos}(D)$. A nonzero point on this bisector ray is $b=\frac{1}{2}\left(\left(D_{._{1}} /\left\|D_{._{1}}\right\|\right)+\left(D_{._{2}} /\left\|D_{\cdot 2}\right\|\right)\right)$, the bisector ray is the ray of this point $b$.

\subsection{Incenter and circumcenter of $\operatorname{Pos}(D)$}

Facets of $\operatorname{Pos}(D)$ are its faces of dimension $n-1$. For $j=1$ to $n$, let $K_{j}=$ $\operatorname{Pos}\left(D_{\cdot 1}, \ldots, D_{\cdot j-1}, D_{\cdot j+1}, \ldots, D_{\cdot n}\right)$ and let $\boldsymbol{H}_{j}$ be the linear hull of $\boldsymbol{K}_{j} . \boldsymbol{K}_{1}, \ldots, \boldsymbol{K}_{n}$ are the facets and $\boldsymbol{H}_{1}, \ldots, \boldsymbol{H}_{n}$ are the facetal hyperplanes for $\operatorname{Pos}(D)$.

The concept of the bisector ray of the angle $\operatorname{Pos}(D)$ when $n=2$, does not directly generalize for $n \geqslant 3$. However, when $n=2$, the important property of a nonzero point on the bisector ray of $\operatorname{Pos}(D)$ is that it is equidistant from each facet of $\operatorname{Pos}(D)$, and this property can be generalized for $n \geqslant 3$. There is a point in the interior of $\operatorname{Pos}(D)$ which is equidistant (say, at a distance of 1) from each of the facetal hyperplanes for $\operatorname{Pos}(D)$, this point is called the incenter for $\operatorname{Pos}(D)$ and its ray is called the incenter ray for $\operatorname{Pos}(D)$. Each point on the incenter ray is equidistant from each of the facetal hyperplanes, and any such point is the center of a sphere tangent to each facet and lying wholly in $\operatorname{Pos}(D)$. Thus the incenter ray projects into the interior of each facet of $\operatorname{Pos}(D)$. See Figure 4.

Let $\beta=\left(\beta_{i j}\right)=D^{-1}$. Then, for $j=1$ to $n$, the facetal hyperplane $\boldsymbol{H}_{j}$ for $\operatorname{Pos}(D)$ is

$$
\boldsymbol{H}_{j}=\left\{x: \beta_{j} . x=0\right\} .
$$

The incenter of $\operatorname{Pos}(D)$ which is at a distance of 1 from each facetal hyperplane of $\operatorname{Pos}(D)$ is $a=D\left(\delta_{1}, \ldots, \delta_{n}\right)^{\mathrm{T}}$ where $\delta_{j}=\left\|\beta_{j}.\right\|$. This point $a$ is clearly in the interior of $\operatorname{Pos}(D)$, and the ray of $a$ is the incenter ray for $\operatorname{Pos}(D)$.

When $n=2$, the incenter ray for $\operatorname{Pos}(D)$ is exactly its bisector ray, and it is a $\mathrm{CP}$-ray. One may be tempted to conjecture that the incenter ray is always a CP-ray for $\operatorname{Pos}(D)$. Unfortunately, this conjecture may be false when $n \geqslant 3$. Let

$$
\hat{D}=\left(\begin{array}{rrr}
-1 & 1 & 20 \\
0 & 3 & 1 \\
0 & 0 & 1
\end{array}\right) \text {. }
$$




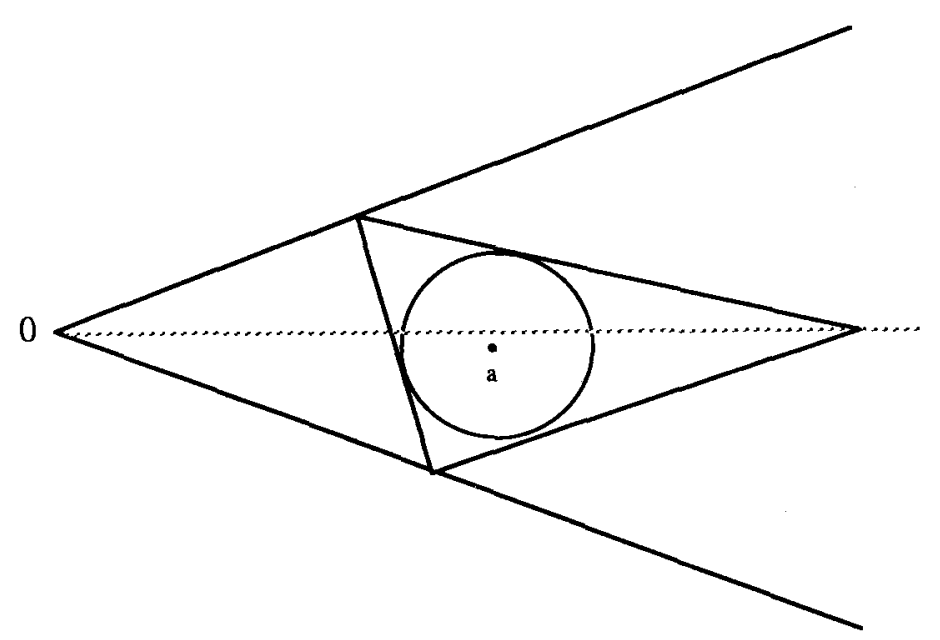

Fig. 4. A simplicial cone in $\mathbb{R}^{3}$ and its incenter $a$.

The incenter ray is not a CP-ray for $\operatorname{Pos}(\hat{D})$. Surprisingly, we found that $\operatorname{Pos}(\hat{D})$ has no CP-point at all. This can be seen from the following. If $b=\left(b_{1}, b_{2}, b_{3}\right)^{\mathrm{T}}$ is a CP-point for $\operatorname{Pos}(\hat{D}), b$ must satisfy

$$
\begin{aligned}
-b_{1} & >0, \\
b_{2} & >0, \\
b_{2}-b_{3} & >0, \\
177 b_{1}-59 b_{2}+10 b_{3} & >0,
\end{aligned}
$$

besides other inequalities imposed by Properties 1 and 2. The first inequality in (3) comes from the requirement that the orthogonal projection of $b$ in the linear hull of $\left\{\hat{D}_{.1}\right\}$ lies in the relative interior of $\operatorname{Pos}\left(\hat{D}_{\cdot 1}\right)$. The second and third inequalities above come from the requirement that $b$ must be in the interior of $\operatorname{Pos}(\hat{D})$, that is, $b=\left(-\alpha_{1}+\alpha_{2}+20 \alpha_{3}, 3 \alpha_{2}+\alpha_{3}, \alpha_{3}\right)^{\mathrm{T}}$ for some $\left(\alpha_{1}, \alpha_{2}, \alpha_{3}\right)>0$. Finally, the orthogonal projection of $b$ in the linear hull of $\left\{\hat{D}_{.2}, \hat{D}_{\cdot 3}\right\}$ is $\gamma_{2} \hat{D}_{.2}+\gamma_{3} \hat{D}_{\cdot 3}$ where $\gamma_{2}=$ $\left(-58 b_{1}+1183 b_{2}-23 b_{3}\right) / 3491$ and $\gamma_{3}=\left(177 b_{1}-59 b_{2}+10 b_{3}\right) / 3491$, and since this point should be in the relative interior of $\operatorname{Pos}\left(\hat{D}_{\cdot 2}, \hat{D}_{\cdot 3}\right)$, we need $\gamma_{2}>0, \gamma_{3}>0$; and $\gamma_{3}>0$ leads to the last inequality in (3). Multiplying the inequalities in (3) by $177,49,10,1$ in that order and summing leads to the inconsistent inequality $0>0$, hence there exists no $b$ satisfying (3), that is no CP-point for $\operatorname{Pos}(\hat{D})$.

At this stage we were quite tempted to conjecture that if $D$ is a square nonsingular matrix such that the incenter ray is not a CP-ray for $\operatorname{Pos}(D)$, then $\operatorname{Pos}(D)$ has no CP-points at all. Unfortunately, this conjecture also turned out to be false. Let

$$
D=\left(\begin{array}{rrr}
1 & -1 & -1 \\
0 & 1 & 1 \\
0 & 0 & 1
\end{array}\right)
$$


When $D$ is the matrix given in (4), the incenter $a=(-1,1+\sqrt{2}, 1)^{\mathrm{T}}$ is not a CP-point for $\operatorname{Pos}(D)$. But $b=(2,8,1)^{\mathrm{T}}$ is a CP-point for $\operatorname{Pos}(D)$.

Given the square nonsingular matrix $D$, the point $d \neq 0$ is said to be the circumcenter of $\operatorname{Pos}(D)$ if

(a) $d$ is equidistant (say, at a distance of 1 ) from each of the generator rays of $\operatorname{Pos}(D)$;

(b) the ray of $d$ makes an acute angle with each of the generator rays of $\operatorname{Pos}(D)$.

The ray of the circumcenter is known as the circumcenter ray for $\operatorname{Pos}(D)$. While the incenter is always contained in the interior of $\operatorname{Pos}(D)$, the circumcenter may not even be in $\operatorname{Pos}(D)$.

The circumcenter ray makes equal acute angles with all the generator rays of $\operatorname{Pos}(D)$ (this provides another equivalent definition of the circumcenter ray for a simplicial cone). From this it can be verified that the circumcenter $\operatorname{ray}$ for $\operatorname{Pos}(D)$ is the ray of $\left(D^{\mathrm{T}}\right)^{-1} \tau$ where $\tau=\left(\tau_{1}, \ldots, \tau_{n}\right)^{\mathrm{T}}, \tau_{j}=\left\|D_{\cdot j}\right\|, j=1$ to $n$. So the circumcenter ray is in the cone $\operatorname{Pos}(D)$ iff $\left(D^{\mathrm{T}} D\right)^{-1} \tau \geqslant 0$.

\subsection{Polar cone}

Let $D$ be a square nonsingular matrix of order $n$. The cone $\left\{y: y^{\mathrm{T}} x \geqslant 0\right.$ for every $x \in \operatorname{Pos}(D)\}$ is known as the polar cone of the cone $\operatorname{Pos}(D)$. Clearly, $y$ is contained in the polar cone of $\operatorname{Pos}(D)$ iff $y^{\mathrm{T}} D_{\cdot j} \geqslant 0$ for all $j=1$ to $n$, that is $D^{\mathrm{T}} y \geqslant 0$. This implies that the polar cone of $\operatorname{Pos}(D)$ is $\operatorname{Pos}\left(\left(D^{\mathrm{T}}\right)^{-1}\right)$.

The Gale-Nikaido theorem [5] states that if $A$ is a P-matrix, then the system $A x \leqslant 0, x \geqslant 0$, has the unique solution $x=0$. Using this and Gordan's theorem of the alternatives $[9,14]$, since $\left(D^{\mathrm{T}} D\right)^{-1}$ is a PD matrix and hence a P-matrix, we conclude that the system $D^{-1}\left(D^{\mathrm{T}}\right)^{-1} x>0, x>0$, has a solution $\bar{x}$. This implies that the point $\left(D^{\mathrm{T}}\right)^{-1} \bar{x}$ is both in the interior of $\operatorname{Pos}(D)$ and the interior of its polar cone. Thus, the interiors of $\operatorname{Pos}(D)$ and its polar cone always have a nonempty intersection. We will prove later (Corollary 1) that every CP-point for $\operatorname{Pos}(D)$ must be an interior point of its polar cone $\operatorname{Pos}\left(\left(D^{T}\right)^{-1}\right)$.

Here are some important properties of the polar cone of $\operatorname{Pos}(D)$. The circumcenter ray for $\operatorname{Pos}(D)$ can be verified to lie always in the polar cone of $\operatorname{Pos}(D)$ by definition.

The circumcenter (incenter) of $\operatorname{Pos}(D)$ is the incenter (circumcenter) of its polar cone.

Let $\beta=D^{-1}$ and let $\boldsymbol{H}_{1}=\left\{x: \beta_{1} . x=0\right\}$ be the facetal hyperplane containing $\operatorname{Pos}\left(D_{\bullet_{2}}, \ldots, D_{\bullet n}\right)$. If $y \notin \boldsymbol{H}_{1}$, the orthogonal projection of $y$ on $\boldsymbol{H}_{1}$ is $\hat{y}=$ $y-\left(1 /\left\|\beta_{1} \cdot\right\|^{2}\right)\left(\beta_{1} \cdot\right)^{\mathrm{T}} \beta_{1} \cdot y$. Since $\beta_{1} . D_{\cdot j}=0$ for all $j=2$ to $n$, it can be verified that if $y^{\mathrm{T}} D \geqslant 0$, then $(\hat{y})^{\mathrm{T}} D_{\cdot j} \geqslant 0$ also for all $j=2$ to $n$. This implies that the orthogonal projection of the polar cone of $\operatorname{Pos}(D)$ on $\boldsymbol{H}_{1}=\mathbf{L H}\left\{D_{\cdot 2}, \ldots, D_{v_{n}}\right\}$ is the polar cone of the face $\operatorname{Pos}\left(D_{._{2}}, \ldots, D_{n_{n}}\right)$. In the same way it can be verified that the orthogonal projection of the polar cone of $\operatorname{Pos}(D)$ on the linear hull of any face of $\operatorname{Pos}(D)$ is the polar cone of that face.

Consider the special case $n=2$. In this case if $\operatorname{Pos}(D)$ is a non-acute angle (see Figure 3) the polar cone $\operatorname{Pos}\left(\left(D^{\mathrm{T}}\right)^{-1}\right) \subset \operatorname{Pos}(D)$, and from Section 3.2 the set of 
CP-points of $\operatorname{Pos}(D)$ is the interior of the polar cone. If $\operatorname{Pos}(D)$ is a non-obtuse angle, $\operatorname{Pos}\left(\left(D^{\mathrm{T}}\right)^{-1}\right) \supset \operatorname{Pos}(D)$, and the set of CP-points for $\operatorname{Pos}(D)$ is its own interior.

\subsection{Dihedral angles and inward normals to facetal hyperplanes}

As before, let $D$ be a square nonsingular matrix of order $n$ and $\beta=D^{-1}$. The hyperplane $\boldsymbol{H}_{j}$ defined in (1) are the facetal hyperplanes for $\operatorname{Pos}(D)$. For $i, j \in \Gamma, i \neq j$, the intersection of the closed half-spaces defined by $\boldsymbol{H}_{\boldsymbol{i}}$ and $\boldsymbol{H}_{j}$ containing the cone $\operatorname{Pos}(D)$ is known as the dihedral angle defined by the pair of facetal hyperplanes $\boldsymbol{H}_{i}$ and $\boldsymbol{H}_{j}$.

Let $g_{\cdot j}=\left(\beta_{j \cdot}\right)^{\mathrm{T}} /\left\|\beta_{j \cdot}\right\|^{2}$. The ray of $g_{\cdot j}$ is the unit normal to the facetal hyperplane $\boldsymbol{H}_{j}$ on the same side of $\boldsymbol{H}_{j}$ as $\operatorname{Pos}(D)$, hence it is known as an inward normal to the facetal hyperplane $\boldsymbol{H}_{j}$. There are $\left(\begin{array}{l}n \\ 2\end{array}\right)$ dihedral angles associated with any simplicial cone in $\mathbb{R}^{n}$. For $i, j \in \Gamma, i \neq j$, a measure of the dihedral angle between $\boldsymbol{H}_{i}, \boldsymbol{H}_{j}$ in radians is

$$
\begin{aligned}
\theta & =\pi-\left(\text { angle between inward normals to } \boldsymbol{H}_{i}, \boldsymbol{H}_{j} \text { in radians }\right) \\
& =\pi-\cos ^{-1}\left(\left(g_{\cdot i}\right)^{\mathrm{T}} g_{\cdot j}\right) .
\end{aligned}
$$

The polar cone of $\operatorname{Pos}(D), \operatorname{Pos}\left(\left(D^{\mathrm{T}}\right)^{-1}\right)$, is a subset of $\operatorname{Pos}(D)$ iff, for every $y \geqslant 0$, $\left(D^{\mathrm{T}}\right)^{-1} y=D x$ for some $x \geqslant 0$, that is, iff $D^{-1}\left(D^{\mathrm{T}}\right)^{-1} y \geqslant 0$ for all $y \geqslant 0$. This holds iff $D^{-1}\left(D^{\mathrm{T}}\right)^{-1}=\beta \beta^{\mathrm{T}} \geqslant 0$, that is, iff all the dihedral angles associated with $\operatorname{Pos}(D)$ are non-acute (i.e., obtuse or right). Similarly, it can be verified that the polar cone of $\operatorname{Pos}(D)$ is in the interior of $\operatorname{Pos}(D)$ iff all the dihedral angles associated with $\operatorname{Pos}(D)$ are strictly obtuse, and in this case the circumcenter ray of $\operatorname{Pos}(D)$ is in its interior.

\subsection{CP-Owning, or CP-lacking simplicial cones}

Given the square nonsingular matrix $D$ of order $n$, we will say that the simplicial cone $\operatorname{Pos}(D)$ is CP-owning (or a CP-owner) if it has at least one CP-point. It is $C P$-lacking (or a CP-lacker) otherwise.

We have already shown that every two dimensional simplicial cone is a CP-owner. But for $n \geqslant 3$, simplicial cones may or may not be CP-owners.

\subsection{CP-Points for lower dimensional cones}

Let $A$ be an $n \times r$ matrix where $r<n$, whose set of column vectors is linearly independent. Then $\operatorname{Pos}(A)$ is an $r$-dimensional cone in $\mathbb{R}^{n}$ which has empty interior. A CP-point for $\operatorname{Pos}(A)$ is defined exactly as before, with the exception that Property 1 now requires the point to be in the relative interior of $\operatorname{Pos}(A)$.

\subsection{Summary of our main results on CP-points}

Let $D$ be a square nonsingular matrix of order $n$. Let $\boldsymbol{K}=\operatorname{Pos}(D)$; and $\boldsymbol{K}^{*}=$ $\operatorname{Pos}\left(\left(D^{\mathrm{T}}\right)\right)^{-1}$, its polar. Let $\boldsymbol{C}$ and $\boldsymbol{C}^{*}$ denote the sets of CP-points of $\boldsymbol{K}$ and $\boldsymbol{K}^{*}$, respectively. As seen in Section 3.2, there are only two possibilities when $n=2$. They are:

(i) $\boldsymbol{K}^{*} \subset \boldsymbol{K}, \boldsymbol{C}=\boldsymbol{C}^{*}=$ interior of $\boldsymbol{K}^{*}$.

(ii) $\boldsymbol{K} \subset \boldsymbol{K}^{*}, \boldsymbol{C}=\boldsymbol{C}^{*}=$ interior of $\boldsymbol{K}$. 
Of course, there are many more possibilities when $n \geqslant 3$. However, our main results on $\mathrm{CP}$-points in the following sections fall into two main streams corresponding to these two possibilities (i) and (ii).

One stream is concerned with the case where the angles between every pair of generator rays of $\boldsymbol{K}$ (i.e., the generator angles of $\boldsymbol{K}$ ) are non-acute, that is, when $M=D^{\mathrm{T}} D$ is a Z-matrix. In this case $\boldsymbol{K}^{*} \subset \boldsymbol{K}$ and $\boldsymbol{C}=\boldsymbol{C}^{*}=\boldsymbol{K}^{*}$. In this case the dihedral angles associated with every face of $\operatorname{Pos}(D)$ are all non-acute.

The second stream is concerned with the case where all the dihedral angles associated with $\boldsymbol{K}$ are non-obtuse. This property is inherited by all the faces of $\boldsymbol{K}$. In this case $M^{-1}=\left(D^{\mathrm{T}} D\right)^{-1}$ is a Z-matrix, and all the generator angles in $K$ are non-obtuse. Here $\boldsymbol{K} \subset \boldsymbol{K}^{*}$ and $\boldsymbol{C}=\boldsymbol{C}^{*}=$ interior of $\boldsymbol{K}$.

\section{Application to LCP}

Let $M=\left(m_{i j}\right)$ be a given real square matrix of order $n$ and $q=\left(q_{j}\right)$ a given column vector in $\mathbb{R}^{n}$. The LCP with data $q, M$, denoted by $(q, M)$ is the problem of finding vectors $w=\left(w_{j}\right), z=\left(z_{j}\right) \in \mathbb{R}^{n}$, satisfying

$$
w-M z=q, \quad w, z \geqslant 0, \quad w^{\mathrm{T}} z=0 .
$$

The LCP is a fundamental problem in mathematical programming, it has been the focus of extensive research over the last 25 years. CP-points came up in a study dealing with the construction of efficient pivotal algorithms for solving certain classes of LCPs. In this section we review this application of CP-points in LCP.

In (5), the pair of variables $\left\{w_{j}, z_{j}\right\}$ is known as the $j$ th complementary pair of variables for $j=1, \ldots, n$. In (5), the column vector associated with $w_{j}$ is $I_{\cdot j}$, and the column vector associated with $z_{j}$ is $-M_{\cdot j}$. The pair $\left\{I_{\cdot j},-M_{\cdot j}\right\}$ is the $j t h$ complementary pair of column vectors in (5). A complementary vector of variables in (5) is a vector $y=\left(y_{1}, \ldots, y_{n}\right)^{\mathrm{T}}$ where $y_{j} \in\left\{w_{j}, z_{j}\right\}$ for each $j=1, \ldots, n$. If $A_{\cdot j}$ is the column in (5) associated with $y_{j}$, the matrix $A=\left(A_{._{1}} \ldots A_{._{n}}\right)$ is known as the complementary matrix associated with $y$. The complementary vector $y$ is said to be a complementary basic vector in (5) if the corresponding complementary matrix is nonsingular. If $y$ is a complementary basic vector associated with the complementary matrix $A$, the complementary basic solution of (5) corresponding to $y$ is given by

$$
\text { all variables in (5) not in } y \text { are }=0, \quad y=A^{-1} q \text {. }
$$

Clearly, the solution in (6) satisfies the complementarity condition $w^{\mathrm{T}} z=0$, because $y$ is a complementary vector. If $A^{-1} q \geqslant 0$, the solution in (6) is a solution of the $\operatorname{LCP}(q, M)$ (it satisfies all the conditions in (5)). It is then said to be a complementary basic feasible solution, and $y$ is said to be a complementary feasible basic vector for (5). The complementary basic feasible solution in (6), and complementary feasible basic vector $y$ are nondegenerate if $A^{-1} q>0$. 
When $M \in P_{n}$, it is well known that the $\operatorname{LCP}(q, M)$ has a unique solution for all $q \in \mathbb{R}^{n}$. Hence for $M \in P_{n}$, if $y$ is a complementary feasible basic vector for the LCP $(q, M)$, which is nondegenerate, it is the unique complementary feasible basic vector for this LCP. Furthermore, there is a unique complementary matrix associated with $y$.

For any $\boldsymbol{J} \subset \Gamma$ the LCP of order $|\boldsymbol{J}|$,

$$
w_{J}-M_{J J} z_{J}=q_{J}, \quad w_{J}, z_{J} \geqslant 0, \quad\left(w_{J}\right)^{\mathrm{T}} z_{J}=0,
$$

which is the LCP $\left(q_{J}, M_{J J}\right)$, is known as the principal subproblem of (5) corresponding to the subset $\boldsymbol{J}$.

One method for solving the LCP $(q, M)$ when $M$ is a P-matrix is the following. Select a column vector $p>0$ in $\mathbb{R}^{n}$. Consider the following parametric LCP, where $\alpha$ is a nonnegative parameter

$$
\begin{array}{cc|c}
w & z & \\
& & \\
\hline I & -M & q+\alpha p
\end{array} \quad w, z \geqslant 0, \quad w^{\mathrm{T}} z=0 .
$$

When $\alpha>0$ is sufficiently large, $w$ is a complementary feasible basic vector for (8), and the method is initiated with this. The method moves through complementary basic vectors for (8), exchanging one basic variable by its complement in each pivot step, in an effort to find a complementary feasible basic vector for (8) for smaller and smaller values of $\alpha$ until it reaches the value 0 . In some general stage, let $\left(w_{\bar{J}}, z_{J}\right)$ be the complementary basic vector for (4) at this stage, for some $\boldsymbol{J} \subset \Gamma$, $\overline{\boldsymbol{J}}=\boldsymbol{\Gamma} \backslash \boldsymbol{J}$. The corresponding basic solution for (8) is

$$
\left(w_{J}, z_{\bar{J}}\right)=0, \quad\left(\begin{array}{c}
z_{J} \\
w_{\bar{J}}
\end{array}\right)=\left(\begin{array}{c}
\bar{q}_{J}+\alpha \bar{p}_{J} \\
\bar{q}_{\bar{J}}+\alpha \bar{p}_{\bar{J}}
\end{array}\right),
$$

where

$$
\left(\bar{q}_{J}, \bar{p}_{J}\right)=-\left(M_{J J}\right)^{-1}\left(q_{J}, p_{J}\right), \quad\left(\bar{q}_{\bar{J}}, \bar{p}_{\bar{J}}\right)=\left(q_{\bar{J}}, p_{\bar{J}}\right)+M_{\bar{J} J}\left(\bar{q}_{J}, \bar{p}_{J}\right) .
$$

The smallest value of $\alpha$ for which the right-hand side of (9) remains $\geqslant 0$ is $\theta$ given by

$$
\theta=\left\{\begin{array}{l}
\text { Maximum }\left\{-\bar{q}_{j} / \bar{p}_{j}: j \text { such that } \bar{p}_{j}>0\right\}, \\
-\infty, \quad \text { if } \bar{p} \leqslant 0 .
\end{array}\right.
$$

If $\theta \leqslant 0,\left(w_{\bar{J}}, z_{J}\right)$ is a complementary feasible basic vector for (8) for $\alpha=0$, that is, a complementary feasible basic vector for (5), terminate. Otherwise, let $r$ be the maximizing index in (10) (or the maximum among these indices, if there is a tie). Perform a pivot step replacing the basic variable from the pair $\left\{w_{r}, z_{r}\right\}$ in the current complementary basic vector by its complement, and continue the method with the new complementary basic vector.

This method is known to solve the $\operatorname{LCP}(q, M)$, when $M$ is a P-matrix, in a finite number of pivot steps [11]. Even though this method is finite, in the worst case, it 
may take up to $2^{n}$ pivot steps before termination $[12,14]$. However, in [17] Pang and Chandrasekaran observed that if the column vector $p$ is selected so that it satisfies the following Property 3 , then the method will terminate after at most $n$ pivot steps, for any $q \in \mathbb{R}^{n}$.

Property 3. $\left(M_{J J}\right)^{-1} p_{J}>0$ for all nonempty $J \subset \Gamma$.

Since $M=\left(m_{i j}\right)$ is a P-matrix, $m_{i i}>0$ for all $i=1$ to $n$. Taking $\boldsymbol{J}=\{i\}$ in Property 3 implies that $p_{i}>0$, hence any vector $p$ satisfying Property 3 is automatically $>0$.

Conditions similar to these have also been discussed earlier by Cottle [2].

If the column vector $p$ satisfies Property 3 , in the above method, when $\left(w_{\bar{J}}, z_{J}\right)$ is the complementary basic vector, in the basic solution $(9), \bar{p}_{j}<0$ for all $j \in J$; and the maximizing index $r$ giving the value of $\theta$ in (10) will not be in $J$. That is, once a $z$-variable becomes basic in the above method, it will stay basic until termination. This guarantees that the method terminates after at most $n$ pivot steps.

\section{PC-Vectors for a P-matrix}

Given a P-matrix $M$ of order $n$, the column vector $p \in \mathbb{R}^{n}$ is said to be a PC-vector for $M$ if it satisfies Property 3 given above. Such a column vector was first defined in the Pang and Chandrasekaran paper [17], and hence the name "PC-vector".

The following Lemmas 2 and 3 and Theorem 1 relating PC-vectors to CP-points follow from the results established in Murty [13].

Lemma 2. Let $M \in P_{n}, p \in \mathbb{R}^{n}$. The column vector $p$ is a PC-vector for $M$ iff the vector composed of the z-variables only, is a nondegenerate complementary feasible basic vector in every principal subproblem of the following $L C P$ :

$$
w-M z=-p, \quad w, z \geqslant 0, \quad w^{\mathrm{T}} z=0 .
$$

Proof. Follows by direct verification.

We will now show that for the class of PD symmetric matrices $M$, PC-vectors correspond to CP-points of a related simplicial cone.

Let $M$ be a PD symmetric matrix of order $n$. Let $D$ denote a real nonsingular square matrix of order $n$ satisfying $D^{\mathrm{T}} D=M$ (for example, the Cholesky factor [15] of $M$ is a candidate for $D$ ). Let $p \in \mathbb{R}^{n}, p>0$, and

$$
b=\left(D^{\mathrm{T}}\right)^{-1} p
$$

Lemma 3. For $J \subset \Gamma, \operatorname{Pos}(D . J)$ is a projection face of $\operatorname{Pos}(D)$ relative to $b$ iff $\left(M_{J J}\right)^{-1} p_{J}>0$, or equivalently, iff $z_{J}$ is the nondegenerate complementary feasible basic vector for the principal subproblem of (11) corresponding to $\mathbf{J}$. 
Proof. If $\boldsymbol{J}=\emptyset$, the result holds by convention. So, assume $\boldsymbol{J} \neq \emptyset$ and let $|\boldsymbol{J}|=s$. The orthogonal projection of $b$ in $\mathbf{L H}\left(D_{\cdot J}\right)$ is $D_{\cdot_{J}} \lambda_{J}$, where $\lambda_{J}$ is the optimum solution of the problem

$$
\begin{array}{ll}
\operatorname{minimize} & \left(b-D_{\cdot J_{J}} \lambda_{J}\right)^{\mathrm{T}}\left(b-D_{._{J}} \lambda_{J}\right) \\
\text { over } & \lambda_{J} \in \mathbb{P}^{s}
\end{array}
$$

which is

$$
\bar{\lambda}_{\boldsymbol{J}}=\left(\boldsymbol{M}_{\boldsymbol{J} J}\right)^{-1}\left(D_{\cdot \boldsymbol{J}}\right)^{\mathrm{T}} \boldsymbol{b} .
$$

So, the orthogonal projection of $b$ in $\mathbf{L H}\left(D_{. J}\right)$, is in the relative interior of the face $\operatorname{Pos}\left(D_{._{J}}\right)$, if $\bar{\lambda}_{J}$ given by (13) is $>0$, that is, iff $\left(M_{J J}\right)^{-1}\left(D_{\cdot J}\right)^{\mathrm{T}} b=\left(M_{J J}\right)^{-1} p_{J}>0$, or equivalently, iff $z_{J}$ is the nondegenerate complementary feasible basic vector for the principal subproblem of (11) corresponding to $J$.

Theorem 1. Let $D$ be a real nonsingular square matrix of order $n$ and $p \in \mathbb{R}^{n}$. Let $M=D^{\mathrm{T}} D, b=\left(D^{\mathrm{T}}\right)^{-1} p$. The vector $p$ is a PC-vector for $M$ iff $b$ is a CP-point for $\operatorname{Pos}(D)$.

Proof. Follows directly from Lemmas 2 and 3.

Corollary 1. Let $D$ be a real nonsingular square matrix of order $n$. Every CP-point for $\operatorname{Pos}(D)$ must be an interior point of the polar cone of $\operatorname{Pos}(D), \operatorname{Pos}\left(\left(D^{\mathrm{T}}\right)^{-1}\right)$.

Proof. Let $b$ be a CP-point for $\operatorname{Pos}(D)$. Then by Theorem $1, p=D^{\mathrm{T}} b$ is a PC-vector for $M=D^{\mathrm{T}} D$, and hence $p=D^{\mathrm{T}} b>0$. Since $b=\left(D^{\mathrm{T}}\right)^{-1} p$, this implies that $b$ is an interior point of $\operatorname{Pos}\left(\left(D^{\mathrm{T}}\right)^{-1}\right)$.

Corollary 1 can also be proved very directly. If $b$ is a CP-point for $\operatorname{Pos}(D)$, each of the generator rays $\operatorname{Pos}\left(D_{\cdot j}\right), j=1$ to $n$, must be a projection face relative to $b$, by definition. This implies that $\left(D_{\cdot j}\right)^{\mathrm{T}} b>0$ for all $j=1$ to $n$, that is, $D^{\mathrm{T}} b>0$, or $b$ must be in the interior of the polar cone of $\operatorname{Pos}(D), \operatorname{Pos}\left(\left(D^{\mathrm{T}}\right)^{-1}\right)$.

Geometrically, Corollary 1 says that every CP-ray for $\operatorname{Pos}(D)$ must make a strict acute angle with each of the generator rays of $\operatorname{Pos}(D)$.

Corollary 2. Let $D$ be a real nonsingular square matrix of order $n$. The set of CP-points for $\operatorname{Pos}(D)$ is a subset of the intersection of the interiors of $\operatorname{Pos}(D)$ and $\operatorname{Pos}\left(\left(D^{T}\right)^{-1}\right)$.

Proof. Follows from the definition and Corollary 1.

Let $D$ be a square nonsingular matrix of order $n$. When $n=2$, in Section 3 we have seen that the set of CP-points of $\operatorname{Pos}(D)$ is always (interior of $\operatorname{Pos}(D)$ ) $\cap$ (interior of $\operatorname{Pos}\left(\left(D^{\mathrm{T}}\right)^{-1}\right)$ ). In Sections 7 and 8 we establish that this result also holds for some special classes of matrices $D$ when $n>2$. Also, when $n=2$, the set of CP-points of $\operatorname{Pos}(D)$ is either the interior of $\operatorname{Pos}(D)$ (when $\operatorname{Pos}(D)$ is an acute or 
right angle), or the interior of $\operatorname{Pos}\left(\left(D^{\mathbf{T}}\right)^{-1}\right)$ (when $\operatorname{Pos}(D)$ is an obtuse angle). In Sections 7 and 8 we derive some necessary and sufficient conditions on $D$, for these properties to hold, when $n>2$.

Theorem 2. Let $M$ be a $P D$ symmetric matrix of order $n$, and let $D$ be a square matrix satisfying $D^{\mathrm{T}} D=M$. There exists a $P C$-vector for $M$ iff $\operatorname{Pos}(D)$ is a CP-owner.

Proof. Follows directly from Theorem 1.

Theorem 3. Let $M$ be a P-matrix of order $n$. The set of all PC-vectors for $M$ is either $\emptyset$ or is an open polyhedral cone.

Proof. A vector $p \in \mathbb{R}^{n}$ is a PC-vector for $M$ iff it is a feasible solution of the system

$$
\left(M_{J J}\right)^{-1} p_{J}>0 \quad \text { for all } \emptyset \neq J \subset \Gamma \text {. }
$$

(14) is a finite system of strict linear inequalities, and its set of feasible solutions is the set of PC-vectors for $M$. Hence, if this set is nonempty, it is an open polyhedral cone.

Theorem 4. Let $D$ be a square nonsingular matrix of order $n$. The set of CP-points for $\operatorname{Pos}(D)$ is either empty, or is an open polyhedral cone.

Proof. Let $M=D^{\mathrm{T}} D$, and let $\Delta$ be the set of PC-vectors for $M$. By Theorem 1 , the set of all CP-points for $\operatorname{Pos}(D)$ is $\left\{x: x=\left(D^{\mathrm{T}}\right)^{-1} p, p \in \Delta\right\}$ and this is an open polyhedral cone when it is nonempty, since the same property holds for $\Delta$.

\section{PC-Vectors for P-matrices of order 2}

Suppose $M=\left(M_{i j}\right)$ is a P-matrix of order 2. Then from the definitions, $p=\left(p_{1}, p_{2}\right)^{\mathrm{T}}$ is a $\mathrm{PC}$-vector for $\boldsymbol{M}$ iff it is feasible to:

$$
\begin{aligned}
m_{22} p_{1}-m_{12} p_{2} & >0, \\
-m_{21} p_{1}+m_{11} p_{2} & >0, \\
p_{1} & >0, \\
p_{2} & >0 .
\end{aligned}
$$

Using Gordan's theorem of the alternatives [9, 14], and the Gale-Nikaido theorem [5], it can be verified that (15) is always feasible. Hence, every P-matrix $M$ of order 2 has a PC-vector $p$, which can be found by solving (15). 


\section{The hereditary feature of these properties}

Let $D$ be a square nonsingular matrix of order $n$.

Theorem 5. If $\operatorname{Pos}(D)$ is a CP-owner, so is every face of $\operatorname{Pos}(D)$.

Proof. Consider the facet $\operatorname{Pos}\left(D_{\cdot_{2}}, \ldots, D_{\cdot n}\right)=K_{1}$ of $\operatorname{Pos}(D)$ and the facetal hyperplane $\boldsymbol{H}_{1}$ containing it. Suppose $b$ is a CP-point for $\operatorname{Pos}(D)$. Let $b^{1}$ be the orthogonal projection of $b$ in $\boldsymbol{H}_{1}$. Since $b$ is a CP-point for $\operatorname{Pos}(D), b^{1}$ is in the relative interior of $\boldsymbol{K}_{1}$. By Pythagoras theorem, $\|x-b\|^{2}=\left\|x-b^{1}\right\|^{2}+\left\|b-b^{1}\right\|^{2}$ for all $x \in \boldsymbol{H}_{1}$. So, if $\boldsymbol{F}$ is any face of $\boldsymbol{K}_{1}$, the orthogonal projections of $b, b^{1}$ in the linear hull of $\boldsymbol{F}$, are the same. So, by the hypothesis, $b^{1}$ is a CP-point for $\boldsymbol{K}_{1}$. A similar proof holds for all facets of $\operatorname{Pos}(D)$, so all facets of $\operatorname{Pos}(D)$ are CP-owners if $\operatorname{Pos}(D)$ is a CP-owner. Repeating this argument, we conclude that all faces of $\operatorname{Pos}(D)$ are CP-owners if $\operatorname{Pos}(D)$ is.

Theorem 6. If every interior point of $\operatorname{Pos}(D)$ is a CP-point for it, then for every face of $\operatorname{Pos}(D)$, every relative interior point is a CP-point.

Proof. Consider the facet $\boldsymbol{K}_{1}=\operatorname{Pos}\left(D_{\cdot 2}, \ldots, D_{\cdot_{n}}\right)$ of $\operatorname{Pos}(D)$. Let $b^{1}$ be any point in the relative interior of $K_{1}$. Erect the inward normal at $b^{1}$ to the facetal hyperplane containing $K_{1}$ and let $b$ be a point on this normal in the interior of $\operatorname{Pos}(D)$. By the hypothesis of the theorem, $b$ is a CP-point for $\operatorname{Pos}(D)$, so by the arguments in the proof of Theorem $5, b^{1}$ is a CP-point for $\boldsymbol{K}_{1}$. So, every point in the relative interior of $K_{1}$ is a CP-point for $\boldsymbol{K}_{1}$. A similar proof holds for all facets of $\operatorname{Pos}(D)$; so, every facet inherits the property that all points in its relative interior are its CP-points. Repeating this argument, we conclude that every relative interior point of any face of $\operatorname{Pos}(D)$ is a CP-point of that face, if every interior point of $\operatorname{Pos}(D)$ is its CP-point.

Theorem 7. Let $M$ be a P-matrix of order $n$. If $M$ has a PC-vector, so does every principal submatrix of $M$.

Proof. Let $p$ be a PC-vector for $M$. Then, for every $\boldsymbol{J} \subset \Gamma$, it follows directly from the definition; that $p_{J}$ is a $\mathrm{PC}$-vector for the principal submatrix $M_{J J}$ of $M$ corresponding to the subset $\boldsymbol{J}$.

\section{CP-Points for $\operatorname{Pos}(D)$ when every pair of generators make a non-acute angle}

Theorem 8. Let $D$ be a square nonsingular matrix of order $n$. If every pair of generators of $\operatorname{Pos}(D)$ make a non-acute (i.e., either obtuse or right) angle, then $\operatorname{Pos}(D)$ is a 
CP-owner, and the set of CP-points of $\operatorname{Pos}(D)$ is the interior of the polar cone of $\operatorname{Pos}(D), \operatorname{Pos}\left(\left(D^{\mathrm{T}}\right)^{-1}\right)$.

Proof. Suppose every pair of generators of $\operatorname{Pos}(D)$ make a non-acute angle. So $\left(D_{\cdot i}\right)^{\mathrm{T}} D_{\cdot j} \leqslant 0$ for all $i \neq j$. Therefore, $M=D^{\mathrm{T}} D$ is a P-matrix which is also a Z-matrix, and hence an M-matrix. By the results in [4] this implies that $M^{-1} \geqslant 0$, and by the same argument $\left(M_{J J}\right)^{-1} \geqslant 0$ for all $\emptyset \neq J \subset \Gamma$. Let $p \in \mathbb{R}^{n}, p>0$. Let $M^{-1} p=y=\left(y_{i}\right)$. Since $M^{-1}$ is also a P-matrix, each of its diagonal elements is $>0$. Since $M^{-1} \geqslant 0$ and has positive diagonal elements and $p>0$, we have $y=M^{-1} p>0$. A similar argument shows that $\left(M_{J J}\right)^{-1} p_{J}>0$ for all $\emptyset \neq J \subset I, p>0$. Hence every $p \in \mathbb{R}^{n}, p>0$, is a $\mathrm{PC}$-vector for $M=D^{\mathrm{T}} D$. Consequently by Theorem 1 , all $b$ in the interior of $\operatorname{Pos}\left(\left(D^{\mathrm{T}}\right)^{-1}\right)$ are CP-points for $\operatorname{Pos}(D)$. By Corollary 1 , this implies that the set of CP-points for $\operatorname{Pos}(D)$ in this case in the interior of $\operatorname{Pos}\left(\left(D^{\mathrm{T}}\right)^{-1}\right)$.

The converse of Theorem 8 , namely that if $D$ is square and nonsingular, and every interior point of the polar cone $\operatorname{Pos}\left(\left(D^{\mathrm{T}}\right)^{-1}\right)$ is a CP-point of $\operatorname{Pos}(D)$, then every pair of generators of $\operatorname{Pos}(D)$ make a non-acute angle, is also true. This is proved along with several other equivalent statements, under Theorem 18 in Section 9.

\section{CP-Points for $\operatorname{Pos}(D)$ when every pair of generators make a non-obtuse angle}

In this section, $D$ is a square nonsingular matrix of order $n$.

In Section 3, we have seen that if $n=2$, and $\operatorname{Pos}(D)$ is an acute or right angle (i.e., $\left.\left(D_{\cdot 1}\right)^{\mathrm{T}} D_{\cdot 2} \geqslant 0\right)$, every interior point of $\operatorname{Pos}(D)$ is a CP-point for it. If $n=3$ and $\left(D_{\cdot i}\right)^{\mathrm{T}}\left(D_{\cdot j}\right) \geqslant 0, i, j=1,2,3$, then any ray interior to $\operatorname{Pos}(D)$ makes acute angles with each generator and hence projects onto the generator rays. Furthermore, we have seen in Section 3.3 that the incenter ray projects to the interior of each facet of $\operatorname{Pos}(D)$. These facts establish that the incenter ray is a CP-ray for such a cone when $n=3$.

These observations might prompt the conjecture that in general a simplicial cone with acute generator angles always has a CP-ray. However, the following example puts that hope to rest.

$$
D=\left(\begin{array}{rrrr}
-1 & 1 & 20 & 0 \\
0 & 3 & 1 & 0 \\
0 & 0 & 1 & 0 \\
5 & 5 & 5 & 5
\end{array}\right)
$$

Even though $\left(D_{\cdot i}\right)^{\mathrm{T}} D_{. j}>0$ for all $i \neq j$, in this matrix $D, \operatorname{Pos}(D)$ is a CP-lacker. This can be seen from the following argument. Let $M=D^{\mathrm{T}} D$. For a vector $p \in \mathbb{R}^{4}$ to be 
a PC-vector for $M$, it must satisfy

$$
\begin{aligned}
-48 p_{2}+35 p_{3} & >0, \\
250 p_{1}+25 p_{2} & -275 p_{4}>0, \\
87275 p_{1}-1450 p_{2}+4425 p_{3}-90250 p_{4} & >0, \\
90250 p_{1}+1475 p_{2}-4575 p_{3}+93359 p_{4} & >0 .
\end{aligned}
$$

The first and the second of these inequalities come from the requirement that $\left(M_{J J}\right)^{-1} p_{J}>0$ for $J=\{2,3\},\{1,2,4\}$ respectively, while the last two are from the same requirement but correspond to $\boldsymbol{J}=\{1,2,3,4\}$. Multiplying the inequalities in this system by $13275,37923,19720,19175$ in that order and summing leads to the inconsistent inequality $0>0$. Thus, no PC-vector exists for $M$, and so by Theorem 1 there is no CP-ray for $\operatorname{Pos}(D)$.

In order to guarantee that $\operatorname{Pos}(D)$ is a CP-owner in this case, we need to impose more conditions on the matrix $D$. Our work on this class of matrices $D$ was motivated by the following conjecture made by Soo Y. Chang.

Conjecture (proved below). Let $\boldsymbol{K}_{j}=\operatorname{Pos}\left(D_{\bullet_{1}}, \ldots, D_{\cdot j-1}, D_{\bullet_{j+1}}, \ldots, D_{\cdot n}\right)$ and $\boldsymbol{H}_{j}$ and facetal hyperplane of $\operatorname{Pos}(D)$ containing $\boldsymbol{K}_{j}$. If for each $j=1$ to $n$, the orthogonal projection of the generator $\operatorname{Pos}\left(D_{. j}\right)$ in $\boldsymbol{H}_{j}$ lies in the relative interior of $\boldsymbol{K}_{j}$, then $\operatorname{Pos}(D)$ is a CP-owner.

While it may not be immediately apparent, we will show that the hypothesis in this conjecture implies that every pair of generators of $D$ make an acute angle, so this conjecture legitimately belongs in this section. We will provide a proof of a stronger form of this conjecture and derive several related results.

For ease of reading, we will summarize the notation for this section. $\boldsymbol{K}_{\boldsymbol{j}}$ and $\boldsymbol{H}_{\boldsymbol{j}}$ are as stated in the above conjecture. $\beta=\left(\beta_{i j}\right)=D^{-1}$. Then,

$$
\begin{aligned}
& \boldsymbol{H}_{j}=\left\{x: \beta_{j} \cdot x=0\right\}, \\
& \operatorname{Pos}(D)=\left\{x: \beta_{j} \cdot x \geqslant 0, j=1 \text { to } n\right\}, \\
& \boldsymbol{K}_{j}=\left\{x: \beta_{i \cdot} x \geqslant 0, i=1 \text { to } n, \quad i \neq j ; \text { and } \beta_{j} \cdot x=0\right\}, \\
& G_{\cdot j}=\left(\beta_{j} \cdot\right)^{\mathrm{T}} /\left\|\beta_{j} \cdot\right\|^{2}, \text { a nonzero point on the inward normal to } \boldsymbol{H}_{j} .
\end{aligned}
$$

Consider the hyperplane $\left\{x: a_{1} x_{1}+\cdots+a_{n} x_{n}=a x=d\right\}$ and let $\bar{x} \in \mathbb{R}^{n}$. The orthogonal projection of $\bar{x}$ in this hyperplane is $\bar{x}+a^{\mathrm{T}}(d-a \bar{x}) /\|a\|^{2}$.

We will now investigate the simplicial cones $\operatorname{Pos}(D)$ satisfying one of these properties. 
Property 4. For each $j=1$ to $n$, the orthogonal projection of the ray $\operatorname{Pos}\left(D_{. j}\right)$ in $\boldsymbol{H}_{j}$ is in the relative interior of $\boldsymbol{K}_{j}$.

Property 5. For each $j=1$ to $n$, the orthogonal projection of the ray $\operatorname{Pos}\left(D_{. j}\right)$ in $\boldsymbol{H}_{j}$ is in $\boldsymbol{K}_{j}$.

Property 6. All the dihedral angles associated with $\operatorname{Pos}(D)$ are acute.

Property 7. All the dihedral angles associated with $\operatorname{Pos}(D)$ are non-obtuse (acute or right).

Property 8. Every interior point of $\operatorname{Pos}(D)$ is a CP-point for it.

Property 9. Every interior point of $\operatorname{Pos}(D)$ is a CP-point for the polar cone $\operatorname{Pos}\left(\left(D^{\mathrm{T}}\right)^{-1}\right)$.

Theorem 9. If Property 4 holds, all the $\left(\begin{array}{l}n \\ 2\end{array}\right)$ dihedral angles associated with $\operatorname{Pos}(D)$ are acute, and conversely.

Proof. The orthogonal projection of $D_{\cdot 1}$ on $H_{1}$ is $D_{\cdot 1}-\left(\beta_{1}\right)^{\mathrm{T}}\left(\beta_{1 \cdot} \cdot D_{\cdot 1}\right) /\left\|\beta_{1} \cdot\right\|^{2}=$ $D_{.1}-G_{.1}$ (since $\beta_{1} . D_{\cdot 1}=1$, as $\beta=D^{-1}$ ). Under Property 4 the orthogonal projection of $D_{\cdot 1}$ on $H_{1}$ is in the relative interior of $K_{1}$, and hence from (19), $\beta_{i \cdot}\left(D_{\cdot 1}-G_{\cdot 1}\right)>0$ for all $i \neq 1$. But $\beta_{i} \cdot D_{.1}=0$ for all $i \neq 1$, since $\beta=D^{-1}$. So $\beta_{i \cdot} G_{\cdot 1}<0$ for all $i \neq 1$, that is, $\left(G_{v_{i}}\right)^{\mathrm{T}} G_{\cdot 1}<0$, for all $i \neq 1$. In the same way, under Property 4 , we have $\left(G_{. i}\right)^{\mathrm{T}} G_{. j}<0$ for all $i \neq j$, that is, all the dihedral angles associated with $\operatorname{Pos}(D)$ are acute. The converse is established by essentially reversing the steps of the proof.

Theorem 10. If Property 5 holds, all the $\left(\begin{array}{c}n \\ 2\end{array}\right)$ dihedral angles associated with $\operatorname{Pos}(D)$ are non-obtuse (i.e., acute or right), and conversely.

Proof. Similar to the proof of Theorem 9.

Thus, Properties 4 and 6 are equivalent. Likewise, Properties 5 and 7 are equivalent.

Lemma 4. Let $\vec{x}$ be any interior point of $\operatorname{Pos}(D)$. If the orthogonal projection of $D .1$ in $\boldsymbol{H}_{1}$ is in the relative interior of $\boldsymbol{K}_{1}$, the orthogonal projection of $\bar{x}$ in $\boldsymbol{H}_{1}$ is also in the relative interior of $\boldsymbol{K}_{1}$.

Proof. Suppose the orthogonal projection of $D_{._{1}}$ in $H_{1}$ is in the relative interior of $K_{1}$. Then, from the proof of Theorem 9, we have, $\beta_{i \cdot}\left(\beta_{1}\right)^{\mathrm{T}}<0$ for all $i \neq 1$.

Now, the orthogonal projection of $\bar{x}$ in $\boldsymbol{H}_{1}$ is $\hat{x}=\bar{x}-\left(\beta_{1} .\right)^{\mathrm{T}}\left(\beta_{1} \cdot \bar{x}\right) /\left\|\beta_{1} \cdot\right\|^{2}$. We have, for $i \neq 1$,

$$
\beta_{i} \cdot \hat{x}=\beta_{i} \cdot \bar{x}+\left(-\beta_{i} \cdot\left(\beta_{1} \cdot\right)^{\mathrm{T}}\right)\left(\beta_{1} \cdot \bar{x}\right) /\left\|\beta_{1} \cdot\right\|^{2}>0
$$


because $\beta_{i} . \bar{x}>0$ (from (18), since $\bar{x}$ is in the interior of $\operatorname{Pos}(D)$ ) for all $i$, and $\left(-\beta_{i \cdot}\left(\beta_{1} \cdot\right)^{\mathrm{T}}\right)>0$ (established above). So, $\hat{x}$ is in the relative interior of $\boldsymbol{K}_{1}$, completing the proof of this lemma.

Theorem 11. If Property 5 holds, then every interior point of $\operatorname{Pos}(D)$ is a CP-point for it, and conversely.

Proof. The proof is essentially similar to the proof of Lemma 4. Suppose Property 5 holds. Let $\bar{x}$ be an interior point of $\operatorname{Pos}(D)$. Let $\boldsymbol{F}$ be a proper face of $\operatorname{Pos}(D)$. So, from (18), there must exist a $\emptyset \neq J \subset \Gamma$ such that

$$
\boldsymbol{F}=\left\{x: \beta_{J} . x=0, \beta_{i} \cdot x \geqslant 0, i \in \Gamma \backslash \boldsymbol{J}\right\} .
$$

Let $|\boldsymbol{J}|=r$. So $\beta_{J}$. is of order $r \times n$ and of full row rank. Hence the matrix $A=\beta_{J} \cdot\left(\beta_{J} \cdot\right)^{\mathrm{T}}$ is PD symmetric. By Theorem 10 , we have $\beta_{i \cdot} \cdot\left(\beta_{\cdot j}\right)^{\mathrm{T}} \leqslant 0$ for all $i \neq j$. This implies that $A$ is a Z-matrix, and hence an M-matrix. By the results in [4], this implies that $A^{-1} \geqslant 0$.

The orthogonal projection of $\bar{x}$ in the linear hull of $F$ is the optimum solution of the problem

$$
\begin{aligned}
& \text { minimize }(x-\bar{x})^{\mathrm{T}}(x-\bar{x}) \\
& \text { subject to } \beta_{J} \cdot x=0,
\end{aligned}
$$

which is $\hat{x}=\bar{x}-\left(\beta_{J} \cdot\right)^{\mathrm{T}} A^{-1} \beta_{J} \cdot \bar{x}$. Let $i \in \Gamma \backslash J$. We have

$$
\beta_{i} \cdot \hat{x}=\beta_{i} \cdot \bar{x}-\beta_{i \cdot}\left(\beta_{J} \cdot\right)^{\mathrm{T}} A^{-1} \beta_{J} \cdot \bar{x}>0,
$$

because $\beta_{i} \cdot \bar{x}>0($ since $\bar{x}$ is in the interior of $\operatorname{Pos}(D)), \beta_{i \cdot}\left(\beta_{J}\right)^{\mathrm{T}} \leqslant 0\left(\right.$ since $\beta_{i \cdot}\left(\beta_{j \cdot}\right)^{\mathrm{T}} \leqslant 0$ for all $j \neq i$ ), $A^{-1} \geqslant 0$ (established above), and $\beta_{J} . \bar{x}>0$ (since $\bar{x}$ is in the interior of $\operatorname{Pos}(D)$ ). So, $\hat{x}$ is in the relative interior of $\boldsymbol{F}$, that is, $\boldsymbol{F}$ is a projection face relative to $\bar{x}$. Since this holds for all faces $F$ of $\operatorname{Pos}(D), \bar{x}$ is a CP-point for $\operatorname{Pos}(D)$. Hence every interior point of $\operatorname{Pos}(D)$ is a CP-point for $\operatorname{Pos}(D)$ in this case.

To prove the converse, suppose every interior point of $\operatorname{Pos}(D)$ is a CP-point for it. Suppose there is a $j$ such that the orthogonal projection of $D_{\cdot j}$ in $\boldsymbol{H}_{j}$ is not in $\boldsymbol{K}_{j}$, say for $j=1$. Since orthogonal projection is a continuous operation, we can find an open ball $\boldsymbol{B}$ containing $D_{._{1}}$ such that the orthogonal projection of every point inside $\boldsymbol{B}$ in $\boldsymbol{H}_{1}$ is outside of $\boldsymbol{K}_{1}$. Since $\boldsymbol{B}$ is an open ball containing $D_{\cdot 1}$, it contains some points from the interior of $\operatorname{Pos}(D)$, and the orthogonal projection of these points are outside $\boldsymbol{K}_{1}$, contradicting the hypothesis. So, if every interior point of $\operatorname{Pos}(D)$ is a CP-point, Property 5 must hold.

Hence, Properties 5 and 8 are equivalent.

We can think of each face of $\operatorname{Pos}(D)$ as being a full dimensional simplicial cone in its linear hull. If $\boldsymbol{F}$ is an $r$-dimensional face of $\operatorname{Pos}(D)$, we can define the $\left(\begin{array}{c}r \\ 2\end{array}\right)$ dihedral angles of $\boldsymbol{F}$ relative to its linear hull, just as we defined the dihedral angles for $\operatorname{Pos}(D)$. 
We will now show that Properties 5 and 7 are facially hereditary, in the sense that if $\operatorname{Pos}(D)$ has the property, then every face of $\operatorname{Pos}(D)$ also has the corresponding property.

Theorem 12. If the simplicial cone $\operatorname{Pos}(D)$ has Property 5 , then every face of $\operatorname{Pos}(D)$ has the corresponding property.

Proof. This follows from Theorems 11 and 6.

Theorem 13. If all the dihedral angles defined by pairs of facets of the simplicial cone $\operatorname{Pos}(D)$ are non-obtuse, every face of $\operatorname{Pos}(D)$ also has the same property.

Proof. Follows directly from Theorems 11, 10 and 12.

Theorem 14. For any simplicial cone $\operatorname{Pos}(D)$, Properties 5, 7 and 8 are equivalent, and these properties are inherited by all faces of $\operatorname{Pos}(D)$.

Proof. Follows from Theorems 10, 11, 12 and 13.

Theorem 15. Property 9 holds iff $\left(D^{\mathrm{T}} D\right)^{-1}$ is a Z-matrix.

Proof. Let $K^{*}=\operatorname{Pos}\left(\left(D^{\mathrm{T}}\right)^{-1}\right)$ be the polar cone of $\operatorname{Pos}(D)$. Define $B=\left(D^{\mathrm{T}}\right)^{-1}$, $M=D^{\mathrm{T}} D, N=B^{\mathrm{T}} B=\left(D^{\mathrm{T}} D\right)^{-1}=M^{-1}$.

Suppose $N=M^{-1}$ is a Z-matrix. Since $N$ is also PD, by the results in [4], $\left(N_{J J}\right)^{-1} \geqslant 0$ for all nonempty $\boldsymbol{J} \subset \Gamma$, and since $\left(N_{J J}\right)^{-1}$ is also PD, each of its diagonal elements is $>0$. Hence, in this case $\left(N_{J J}\right)^{-1} q_{J}>0$ for all $q \in \mathbb{R}^{n}, q>0$, and nonempty $J \subset \Gamma$. Hence, by Theorem $1,\left(B^{\mathrm{T}}\right)^{-1} q=D q$ is a CP-point for $\boldsymbol{K}^{*}$ for all $q>0$, that is every interior point of $\operatorname{Pos}(D)$ is a CP-point for the polar cone $K^{*}$ in this case.

Suppose $D q$ is a CP-point for $\boldsymbol{K}^{*}$ for all $q>0$. Then by Theorem 1 we must have

$$
\left(N_{J J}\right)^{-1} q_{J}>0 \text { for all nonempty } J \subset \Gamma \text { and all } q>0 .
$$

In particular $N^{-1} q>0$ for every $q>0$. Let $1 \leqslant j \leqslant n$. Define $q(\varepsilon)=\left(q_{t}(\varepsilon): t=1\right.$ to $n$ ) where $q_{t}(\varepsilon)=\varepsilon$ for $t \neq j$, and 1 for $t=j$. Since $N^{-1} q(\varepsilon)>0$ for all $\varepsilon>0$, by making $\varepsilon$ tend to zero through positive values, we conclude that $\left(N^{-1}\right)_{\cdot j} \geqslant 0$. Hence $N^{-1} \geqslant 0$.

Using the same argument, we conclude that (22) implies that $\left(N_{J J}\right)^{-1} \geqslant 0$ for every nonempty $J \subset \Gamma$. Now consider $J_{1}=\{1,2\}$. Then denoting the $(i, j)$ th element in the matrix $N$ by $n_{i j}$, we have

$$
N_{J_{1} J_{1}}=\left(\begin{array}{ll}
n_{11} & n_{12} \\
n_{21} & n_{22}
\end{array}\right) .
$$

Since $N=B^{\mathrm{T}} B$ is symmetric, $n_{12}=n_{21}$, and denote their common value by $\gamma$. Also, $N$ is PD, hence, $n_{11}>0, n_{22}>0$ and $n_{11} n_{22}-\gamma^{2}>0$. Using these and $\left(N_{J_{1} J_{1}}\right)^{-1} \geqslant 0$, 
we conclude that $\gamma \leqslant 0$. By the same argument we can show that all off-diagonal elements of $N$ are $\leqslant 0$, that is $N=\left(D^{\mathrm{T}} D\right)^{-1}$ is a Z-matrix, in this case, completing the proof.

Corollary 3. Let $A$ be a P-matrix of order $n$. It is a $Z$-matrix iff $\left(A_{J J}\right)^{-1} \geqslant 0$ for all nonempty $\mathrm{J} \subset \Gamma$.

Proof. Follows from the results in [4], and using the argument made in the proof of Theorem 15.

We will now explain the relationship of these properties to the condition mentioned in the heading of this section, and the role that $Z$-matrices play in these properties. From the results in this section, we see that the basic condition for Properties 5 or 7 or 8 to hold is that

$$
\beta_{i \cdot}\left(\beta_{j}\right)^{\mathrm{T}} \leqslant 0 \text { for all } i \neq j,
$$

that is, that $\beta \beta^{\mathrm{T}}$ is a $\mathrm{Z}$-matrix. Since $\beta$ is nonsingular, this is equivalent to requiring that $\beta \beta^{\mathrm{T}}$ be an M-matrix. But $\beta \beta^{\mathrm{T}}=\left(D^{-1}\right)\left(D^{-1}\right)^{\mathrm{T}}=\left(D^{\mathrm{T}} D\right)^{-1}=M^{-1}$ where $M=$ $D^{\mathrm{T}} D$. So, if (23) holds, $\left(D^{\mathrm{T}} D\right)^{-1}$ is an M-matrix, and by the results in [4], $D^{\mathrm{T}} D \geqslant 0$, in other words, every pair of generators for $\operatorname{Pos}(D)$ makes a non-obtuse angle. Hence all the results in this section relate to a subclass of simplicial cones in which every pair of generators make a non-obtuse angle.

One noteworthy feature. Let $D^{\mathrm{T}} D=M$. In Section 7 we dealt with the case where $M$ is a $Z$-matrix. In this section 8 we are dealing with the case where $M^{-1}$ is a $Z$-matrix. We now provide a theorem which summarizes this section.

Theorem 16. Every interior point of $\operatorname{Pos}(D)$ is a $C P$-point for it, iff $\left(D^{\mathrm{T}} D\right)^{-1}$ is a $Z$-matrix. Also, Properties 5, 7, 8 and 9, and the condition that $\left(D^{\mathrm{T}} D\right)^{-1}$ is a Z-matrix, are all equivalent.

Proof. Requiring $\left(D^{\mathrm{T}} D\right)^{-1}$ to be a $Z$-matrix is equivalent to requiring Property 7 as explained above. So, these results follow from Theorems 14 and 15.

\section{Generalization of the concept of regularity of a simplicial cone}

In classical geometry, a simplex is called a regular simplex if all its edges are of equal length, and the simplicial cone $\operatorname{Pos}(D)$ is called a regular simplicial cone if the convex hull of $\left\{D_{. j} /\left\|D_{. j}\right\|: j=1\right.$ to $\left.n\right\}$ is a regular simplex. Property 8 of course holds for regular simplicial cones, and this is of principal interest when referring to regularity in simplicial cones. So, the conditions given in Theorem 16 (namely that $\left(D^{\mathrm{T}} D\right)^{-1}$ should be a Z-matrix ) provide a proper generalization of the traditional concept of regularity in simplicial cones. It identifies the class of simplicial cones satisfying Property 8. 


\section{Simplicial cones $\operatorname{Pos}(D)$ with all dihedral angles non-acute}

Let $D$ be a square nonsingular matrix of order $n$.

Theorem 17. If $n=3$, and all the dihedral angles associated with $\operatorname{Pos}(D)$ are non-acute, then the circumcenter ray of $\operatorname{Pos}(D)$ is a CP-ray for it.

Proof. From the definition of the dihedral angles associated with $\operatorname{Pos}(D)$ (Section 3), we know that they are all non-acute iff

$$
\left(D^{\mathrm{T}} D\right)^{-1} \geqslant 0 \text {. }
$$

Let $\tau=\left(\tau_{1}, \ldots, \tau_{n}\right)^{\mathrm{T}}$ where $\tau_{j}=\left\|D_{. j}\right\|, j=1$ to $n$, and

$$
b=\left(D^{\mathrm{T}}\right)^{-1} \tau \text {. }
$$

The ray of $b$ is the circumcenter ray for $\operatorname{Pos}(D)$. Since $D^{-1} b=\left(D^{\top} D\right)^{-1} \tau>0$ (because $\tau>0,(24)$, and since $\left(D^{\mathrm{T}} D\right)^{-1}$ is a PD symmetric matrix, its main diagonal is $>0), b$ is in the interior of $\operatorname{Pos}(D)$.

Let $M=\left(m_{i j}\right)=D^{\mathrm{T}} D$. We will now show that

$$
\left(M_{J J}\right)^{-1} \tau_{J}>0 \text { for all } J \subset\{1,2,3\} .
$$

Since $M^{-1} \tau=D^{-1} b>0$, we know that (26) holds for $\boldsymbol{J}=\{1,2,3\}$. When $\boldsymbol{J}=\{1,2\}$, it can be verified that $\left(M_{J J}\right)^{-1} \tau_{J}=\left(\tau_{2}, \tau_{1}\right)^{\mathrm{T}} /\left(\tau_{1} \tau_{2}+\left(D_{.1}\right)^{\mathrm{T}} D_{\cdot 2}\right)>0$ because $\tau_{1}$ and $\tau_{2}$ are $>0$ and $\tau_{1} \tau_{2}+\left(D_{.1}\right)^{\mathrm{T}} D_{.2}>0$ by Cauchy-Schwartz inequality, so (26) holds. By symmetry, (25) holds whenever $|\boldsymbol{J}|=2$. When $\boldsymbol{J}=\{j\},\left(M_{J J}\right)^{-1} \tau_{J}=\left(1 / \tau_{j}\right)>0$ for all $j=1,2,3$, so (26) holds. Hence $\tau$ is a PC-vector for $M$, and by Theorem $1, b=\left(D^{\mathrm{T}}\right)^{-1} \tau$ is a CP-point for $\operatorname{Pos}(D)$. So the circumcenter ray is a CP-ray for $\operatorname{Pos}(D)$ in this case.

For any $n$, if all the dihedral angles associated with $\operatorname{Pos}(D)$ are non-acute, (24) holds. Under (24), the circumcenter ray for $\operatorname{Pos}(D)$ is an interior ray for $\operatorname{Pos}(D)$. Unfortunately, under these conditions, the circumcenter ray is not guaranteed to be a CP-ray if $n \geqslant 4$. Consider the following matrix $D$ of order 4 :

$$
D=\left(\begin{array}{rrrr}
-1 & 1 & 20 & -20 \\
0 & 3 & 1 & -4 \\
0 & 0 & 1 & -1 \\
0 & 0 & 0 & \frac{1}{3}
\end{array}\right)
$$

It can be verified that this matrix satisfies (24), so all the dihedral angles associated with its pos cone are non-acute. Yet, this cone $\operatorname{Pos}(D)$, has no CP-point. This follows from Theorem 5 since its face $\operatorname{Pos}\left(D_{.1}, D_{\cdot 2}, D_{\cdot 3}\right)$ has no CP-point (in its linear hull 
this face $\operatorname{Pos}\left(D_{\cdot 1}, D_{\cdot 2}, D_{\cdot 3}\right)$ is the same as the cone $\operatorname{Pos}(\hat{D})$ where $\hat{D}$ is the matrix given in (2), and it was shown in Section 3.3 that $\operatorname{Pos}(\hat{D})$ is a CP-lacker).

Thus, when $n \geqslant 4$, to guarantee that CP-point exists for a simplicial cone $\operatorname{Pos}(D)$, condition (24) is not adequate by itself, we need to impose more conditions. This leads us to the next theorem, which includes the converse of Theorem 8 in Section 7 , and provides several other equivalent results.

Theorem 18. Let $D$ be a real square nonsingular matrix of order $n$. The following statements are equivalent:

(i) Every pair of generators of $\operatorname{Pos}(D)$ make a non-acute angle, or equivalently $M=D^{\mathrm{T}} D$ is a Z-matrix.

(ii) Every point in the interior of the polar cone, $\operatorname{Pos}\left(\left(D^{\mathrm{T}}\right)^{-1}\right)$ is a CP-point for $\operatorname{Pos}(D)$.

(iii) Every $p \in \mathbb{R}^{n}, p>0$, is a $P C$-vector for $M=D^{\mathrm{T}} D$.

(iv) Every face of $\operatorname{Pos}(D)$ satisfies the property that all the dihedral angles associated with it are non-acute.

(v) Every interior point of the polar cone $\operatorname{Pos}\left(\left(D^{\mathrm{T}}\right)^{-1}\right)$ is a CP-point for it.

Proof. (ii) and (iii) are equivalent by Theorem 1. By Theorem 8, (i) implies (ii). Hence (i) implies (iii).

We will now prove that (iii) implies (i). Assume that (iii) holds. By the definition of a PC-vector, we have $\left(M_{J J}\right)^{-1} p_{J}>0$ for every $p>0$ and every nonempty $J \subset \Gamma$. Using the argument made in the proof of Theorem 15, this implies that $M$ is a Z-matrix, which is equivalent to the statement that every pair of generators of $\operatorname{Pos}(D)$ make a non-acute angle. Hence (iii) implies (i).

Suppose (iv) holds. Let $M=D^{\mathrm{T}} D$. Since all the dihedral angles associated with $\operatorname{Pos}(D)$ are non-acute, by the definition of dihedral angles, $\left(D^{-1}\right)\left(D^{-1}\right)^{\mathrm{T}} \geqslant 0$ that is, $M^{-1} \geqslant 0$. In the same manner, for any $\emptyset \neq J \subset \Gamma$, if the dihedral angles associated with the face $\operatorname{Pos}\left(D_{. J}\right)$ of $\operatorname{Pos}(D)$ are non-acute, it can be shown that $\left(M_{J J}\right)^{-1}=$ $\left((D . J)^{\mathrm{T}} D_{. J}\right)^{-1} \geqslant 0$. Hence $\left(M_{J J}\right)^{-1} \geqslant 0$ for all $\boldsymbol{J} \subset \Gamma$, and hence (iii) holds. Conversely, if (iii) holds, for every $J \subset \Gamma$, we have, $\left(M_{J J}\right)^{-1} p_{J}>0$ for all $p_{J}>0$, this implies that $\left(M_{J I}\right)^{-1} \geqslant 0$ by the argument given above, which in turn implies that the dihedral angles associated with the face of $\operatorname{Pos}(D)$ corresponding to $\boldsymbol{J}$ are all non-acute. Hence (iv) holds if (iii) does. Thus, (iii) and (iv) are equivalent.

Assume that (i) holds. Then $D^{\mathrm{T}} D$ is a $Z$-matrix. Let $B=\left(D^{\mathrm{T}}\right)^{-1}$. Therefore $D^{\mathrm{T}} D=\left(B^{\mathrm{T}} B\right)^{-1}$, hence $\left(B^{\mathrm{T}} B\right)^{-1}$ is a Z-matrix. This implies that all the dihedral angles associated with $\operatorname{Pos}(B)=$ polar cone of $\operatorname{Pos}(D)$, are non-obtuse, and hence by the results in Section 8 every interior point of the polar cone is a CP-point for it, thus (v) holds. Now, if (v) holds, by applying Theorem 16 to the polar cone we conclude that $\left(\left(\left(D^{\mathrm{T}}\right)^{-1}\right)^{\mathrm{T}}\left(D^{\mathrm{T}}\right)^{-1}\right)^{-1}=D^{\mathrm{T}} D$ must be a Z-matrix, so (i) holds. Thus (i) and (v) are equivalent.

Hence conditions (i) to (v) are all mutually equivalent. 
As stated in the summary in Section 3.8, the results in Section 7 and this section correspond to the case where the polar cone $\operatorname{Pos}\left(\left(D^{\mathrm{T}}\right)^{-1}\right) \subset \operatorname{Pos}(D)$, and the sets of CP-points of $\operatorname{Pos}(D)$ and $\operatorname{Pos}\left(\left(D^{\mathrm{T}}\right)^{-1}\right)$ are both the same as the interior of the polar cone. The results in Section 8 correspond to the case where $\operatorname{Pos}(D)$ is a subset of the polar cone, and the sets of CP-points of $\operatorname{Pos}(D)$ and its polar cone are both the same as the interior of $\operatorname{Pos}(D)$.

\section{Open questions}

Given a rational square nonsingular matrix $D$ of order $n$ and a rational point $b$ in the interior of $\operatorname{Pos}(D), b$ has to satisfy Property 2 of Section 3 to be a CP-point for $\operatorname{Pos}(D)$. Property 2 consists of $2^{n}-2$ sets of conditions. It is not known whether there is a polynomially bounded algorithm for checking Property 2 .

J.S. Pang posed the following question: "What is the class of real nonsingular matrices $D$ of order $n$, for which the set of CP-points of $K=\operatorname{Pos}(D)$ is the interior of $\boldsymbol{K} \cap \boldsymbol{K}^{*}$, where $\boldsymbol{K}^{*}$ is the polar cone of $\boldsymbol{K}$ ?"

A mathematical characterization for this class is easy to provide using Theorem 1 . Denoting $D^{\mathrm{T}} D$ by $M$, this is exactly the class of all matrices $D$ for which

$$
p \in \mathbb{R}^{n}, p>0 \text { and } M^{-1} p>0 \text { implies } \quad\left(M_{J J}\right)^{-1} p_{J}>0 \text { for all nonempty } \boldsymbol{J} \subset \Gamma \text {. }
$$

From the results of Sections 7, 8 and 9, we know that this class contains the matrices all of whose generator angles are non-acute, and the matrices all of whose dihedral angles are non-obtuse. It is not known whether this class contains any matrices other than these two streams.

Another interesting problem is the following: if a simplicial cone is a CP-owner, is its polar cone also a CP-owner? A related question is whether the sets of CP-points for a simplicial cone and its polar are the same. This question has been settled in the affirmative using the results from [10].

Let $D$ be a real square nonsingular matrix of order $n$, and let $C$ be the set of CP-points of $\operatorname{Pos}(D)$. We have shown that if $C \neq \emptyset$, it is an open polyhedral cone defined by a system consisting of a large number of constraints ( $2^{n}$ sets of constraints). In the earlier version of this paper, we conjectured that whenever $C \neq \emptyset$, it is a simplicial cone. This conjecture has been settled in the affirmative by Morris Jr. and Lawrence in their recent paper [10].

\section{A generalization}

In this section, we will discuss a generalization of the properties of being a CP-point or a PC-vector. Here, $\mathbb{R}^{n \times n}$ denotes real square matrices of order $n$, which may or may not be nonsingular. We now state two properties. 
Property 10. The pair $(D, q)$, where $D \in \mathbb{R}^{n \times n}$ and $q \in \mathbb{R}^{n}$, is said to have this property if for each nonempty $J \subset \Gamma$ the orthogonal projection of $q$ onto the subspace $\mathbf{L H}\left(D_{. J}\right)$ lies in the relative interior of the cone $\operatorname{Pos}\left(D_{. J}\right)$.

Property 11. The pair $(M, q)$, where $M \in \mathbb{R}^{n \times n}$ and $q \in \mathbb{B}^{n}$, is said to have this property if for each nonempty $J \subset \Gamma$, the linear complementarity problem $\left(-q_{J}, M_{J J}\right)$ has a solution $\left(\tilde{w}_{J}, \tilde{z}_{J}\right)$ with $\tilde{z}_{J}>0$.

Note that the definition of Property 10 is minimal in the sense that every subset $J \subset \Gamma$ must be checked, as the following examples show. The listed $D$ and $q$ have the projection property for every subset of $\{1,2,3\}$ except the ones listed:

$$
\begin{aligned}
& D=\left(\begin{array}{lll}
3 & 2 & 4 \\
2 & 3 & 4 \\
0 & 0 & 1
\end{array}\right), \quad q=\left(\begin{array}{r}
10 \\
10 \\
-1
\end{array}\right), \quad J=\{1,2,3\}, \\
& D=\left(\begin{array}{lll}
0 & 1 & 4 \\
1 & 5 & 4 \\
0 & 0 & 1
\end{array}\right), \quad q=\left(\begin{array}{r}
13 \\
18 \\
3
\end{array}\right), \quad J=\{1,2\}, \\
& D=\left(\begin{array}{rrr}
1 & -1 & -10 \\
1 & 1 & 10 \\
0 & 0 & 1
\end{array}\right), \quad q=\left(\begin{array}{r}
-1 \\
100 \\
1
\end{array}\right), \quad J=\{1\} .
\end{aligned}
$$

Notice that in Property $10, D$ is not required to be nonsingular. Likewise in Property $11, M$ may not be nonsingular. We define

$$
\begin{gathered}
\mathrm{P} 10_{n}=\left\{D \in \mathbb{R}^{n \times n}: \text { there exists at least one } q \in \mathbb{R}^{n}\right. \\
\text { such that }(D, q) \text { satisfies Property } 10\}, \\
\mathrm{P} 11_{n}=\left\{M \in \mathbb{R}^{n \times n}: \begin{array}{l}
\text { there exists at least one } q \in \mathbb{R}^{n} \\
\text { such that }(M, q) \text { satisfies Property } 11\} .
\end{array}\right.
\end{gathered}
$$

By our earlier results, if $D \in \mathbb{R}^{n \times n}$ is nonsingular, $(D, q)$ has Property 10 iff $\left(D^{\mathrm{T}} D, D^{\mathrm{T}} q\right)$ has Property 11.

\section{Convexity results}

Proposition 1. For $D \in \mathbb{R}^{n \times n}$ the set of $q \in \mathbb{R}^{n}$ such that $(D, q)$ has Property 10 is convex and open.

Proof. For every subset $\emptyset \neq J \subset \Gamma$, the set of points $q$ for which the projection of $q$ onto the linear hull of $D_{. J}$ is in the relative interior of $\operatorname{Pos}\left(D_{. J}\right)$ is the interior of an $n$-dimensional polyhedral cone (it is the set of points of the form $x+y$, where $x$ is any point in the relative interior of $\operatorname{Pos}(D . J)$ and $y$ is any point in the orthogonal complement of the linear hull of $D . J)$. The set of $q$ such that $(D, q)$ has Property 10 is then the intersection of a finite number of these, and hence is convex and open. 
Observe that for $n \geqslant 2$, the set $P_{n}$ is not convex. Let

$$
M^{1}=\left(\begin{array}{rr}
1 & -15 \\
1 & 2
\end{array}\right), \quad M^{2}=\left(\begin{array}{rr}
1 & 1 \\
-15 & 2
\end{array}\right) \text {. }
$$

$M^{1}$ and $M^{2}$ are P-matrices, but $\frac{1}{2}\left(M^{1}+M^{2}\right)$ is not.

Since $P_{n}$ is not convex, it turns out that for $n \geqslant 2$ and fixed $q \in \mathbb{R}^{n}$, the set of matrices $M \in P_{n} \cap{\mathrm{P} 11_{n}}_{\text {such }}$ that $(M, q)$ has Property 11 is not necessarily convex.

Conjecture. For fixed $q \in \mathbb{R}^{n}$, the set of PD symmetric $M \in \mathbb{R}^{n \times n}$ such that $(M, q)$ has Property 11 is convex.

Definition. A set $\Delta \subset \mathbb{R}^{n}$ is starlike if there exists a point $c \in \Delta$ such that for any point $p \in \Delta$ the line segment $\overline{c p}$ also lies in $\Delta$. The set of points $c \in \Delta$ with this property is called the kernel of $\Delta$.

Proposition 2 (Howe and Stone [6]). The set $P_{n}$ is starlike with the identity matrix $I$ in its kernel.

Proof. Let $M \in P_{n}$ and $0 \leqslant \alpha \leqslant 1$. A characterization of $P_{n}$ is that $M \in P_{n}$ if and only if the real eigenvalues of every principal submatrix of $M$ are positive $[4,18]$. If $\lambda>0$ is an eigenvalue of a principal submatrix $M_{J J}$, then $(1-\alpha)+\alpha \lambda>0$ is an eigenvalue of $[(1-\alpha) I+\alpha M]_{J J}$. Therefore the real eigenvalues of every principal submatrix of $(1-\alpha) I+\alpha M$, for $0 \leqslant \alpha \leqslant 1$, are positive, and $(1-\alpha) I+\alpha M \in P_{n}$ for $0 \leqslant \alpha \leqslant 1$.

Proposition 3. For fixed $q \in \mathbb{R}^{n}, q>0$, the set of matrices $M \in P_{n}$ such that $(M, q)$ has Property 11 is starlike with I in its kernel.

Proof. Let $(M, q)$ have Property 11 , where $M \in P_{n}, q \in \mathbb{R}^{n}, q>0$. It suffices to prove that $((1-\lambda) M+\lambda I, q)$ also has Property 11 , for $0 \leqslant \lambda \leqslant 1$. The proof of this is by induction on $n$. Since $(M, q)$ and $(I, q)$ have Property 11 , consider only $0<\lambda<1$. The result is trivial for $n=1$. Assume it holds for P-matrices of order $<n$. Property 11 for $(M, q)$ means that $M_{J J}^{-1} q_{J}>0$ for every subset $J, \emptyset \neq J \subset \Gamma$. Since Property 11 is hereditary with respect to principal submatrices, the induction hypothesis yields that $\left[(1-\lambda) M_{J \cdot J}+\lambda I_{J J}\right]^{-1} q_{J}>0$ for every subset $J, \emptyset \neq J \subset \Gamma$, of cardinality $|J|<n$. Thus, all that remains is to prove $[(1-\lambda) M+\lambda I]^{-1} q>0$.

The $k$ th component of $z=[(1-\lambda) M+\lambda I]^{-1} q$ is, by Cramer's rules,

$$
z_{k}=\frac{\operatorname{det}\left([(1-\lambda) M+\lambda I]_{\cdot\{1, \ldots, k-1\}}: q:[(1-\lambda) M+\lambda I]_{\cdot\{k+1, \ldots, n\}}\right)}{\operatorname{det}[(1-\lambda) M+\lambda I]} .
$$

Note that the denominator is positive since $(1-\lambda) M+\lambda I$ is also a P-matrix. The determinant is a multilinear form, and the numerator expands into a sum of 
determinants of the form $\operatorname{det} A$, where $A_{\bullet_{i}}=(1-\lambda) M_{\bullet_{i}}$ or $A_{\bullet_{i}}=\lambda I_{v_{i}}$ for $i \neq k$, and $A_{\cdot k}=q$. Let $\boldsymbol{J}=\left\{j_{1}, \ldots, k, \ldots, j_{r}\right\}$ be the indices such that $A_{._{i}}=\lambda I_{\cdot i}$ for $i \notin J$. Then

$$
\operatorname{det} A=\lambda^{n-|\boldsymbol{J}|}(1-\lambda)^{|\boldsymbol{J}|-1}\left(M_{J J}^{-1} q_{J}\right)_{k} \operatorname{det} M_{J \boldsymbol{J}}>0
$$

since $0 \leqslant \lambda \leqslant 1,(M, q)$ has Property 11 and $M$ is a P-matrix. (The subscript $k$ here refers to the element $k$ in the index set $\boldsymbol{J}=\left\{j_{1}, \ldots, k, \ldots, j_{r}\right\}$.) Therefore the numerator in (27) is positive, and so $[(1-\lambda) M+\lambda I]^{-1} q>0$, which completes the induction step.

Note that $Z_{n}$ is convex but $M_{n}$ is not. Let

$$
M^{3}=\left(\begin{array}{rr}
3 & -16 \\
-2 & 11
\end{array}\right), \quad M^{4}=\left(\begin{array}{rr}
3 & -2 \\
-16 & 11
\end{array}\right) \text {. }
$$

$M^{3}$ and $M^{4}$ are $M$-matrices, but $\frac{1}{2}\left(M^{3}+M^{4}\right)$ is not.

The following theorem from Fiedler and Ptak [4] will be useful.

Theorem 19. Let $A, B \in Z_{n}$, all the real eigenvalues of $A$ be positive, and $A \leqslant B$. Then

(1) $A^{-1} \geqslant B^{-1} \geqslant 0$;

(2) all the real eigenvalues of $B$ are positive;

(3) $\operatorname{det} B \geqslant \operatorname{det} A>0$.

Proposition 4. The set $M_{n}$ is starlike with the identity matrix I in its kernel.

Proof. Let $M \in M_{n}=Z_{n} \cap P_{n}$. It is straightforward to verify that $(1-\lambda) I+\lambda M$ for $0 \leqslant \lambda \leqslant 1$ is in both $Z_{n}$ and $P_{n}$.

Proposition 5. For any $M \in M_{n}$ and $q \in \mathbb{R}^{n}, q>0$, the pair $(M, q)$ has Property 11.

Proof. Let $M \in M_{n}, q \in \mathbb{R}_{n}, q>0$ and $\emptyset \neq J \subset I$. Then $M_{J J}$ is also an M-matrix, hence a $Z$-matrix with positive real eigenvalues. By Theorem 19 above, $M_{J J}^{-1} \geqslant$ $\left(\operatorname{diag}\left(M_{J J}\right)^{-1} \geqslant 0\right.$ is nonnegative with positive diagonal elements, and therefore $M_{J J}^{-1} q_{J}>0$. This proves Property 11 for the pair $(M, q)$.

\section{Generalization of the class $M_{n}$}

M-matrices have many nice properties with respect to the linear complementarity problem. However, they have more structure than is really needed for linear complementarity proofs. We argue that $\mathrm{P} 1_{n} \cap P_{n}$ is the natural generalization of $M_{n}$, and that $\mathrm{P} 11_{n} \cap P_{n}$ contains the essence of the class $M_{n}$ as far as linear complementarity is concerned.

Both $M_{n}$ and $\mathrm{P} 11_{n} \cap P_{n}$ are nonconvex, both are starlike from $I$. When $M \in M_{n}$, for every $q>0,(M, q)$ has Property 11 ; whereas for $M \in \mathrm{P} 11_{n} \cap P_{n}$, there exists a $q \in \mathbb{R}^{n}, q>0$, such that $(M, q)$ has Property 11 . When $M \in M_{n}$, for all $\emptyset \neq J \subset \Gamma$ and every $q \in \mathbb{R}^{n}, q>0,\left(M_{J J}\right)^{-1} q_{J}>0$; whereas, for $M \in \mathbf{P} 11_{n} \cap P_{n}$, there exists a $q \in \mathbb{R}^{n}$, 
$q>0$ such that for all $\emptyset \neq J \subset \Gamma,\left(M_{J J}\right)^{-1} q_{J}>0$. Similarly, when $M \in M_{n}$, the statement "for any $q \in \mathbb{R}^{n}$, the LCP $(q, M)$ can be solved with at most $n$ complementary pivot steps via the parametric LCP $(q+\alpha p, M)$, is true for every $p>0$ " whereas, for $M \in \mathrm{P} 11_{n} \cap P_{n}$, there exists a $p>0$ such that the above statement within quotes is true.

\section{Acknowledgement}

We thank the referees for their thorough reports. The simple proof of Proposition 1 is due to a referee. This referee has also brought the reference [6] where Proposition 2 has already appeared, to our attention.

\section{References}

[1] S.Y. Chang and K.G. Murty, "The Steepest descent gravitational method for linear programming," Discrete Applied Mathematics, to appear.

[2] R.W. Cottle, "Monotone solutions of the parametric linear complementarity problem," Mathematical Programming 3 (1972) 210-224.

[3] B.C. Eaves, F.J. Gould, H.O. Peitgen and M.J. Todd, eds., Homotopy Methods and Global Convergence (Plenum, New York, 1983).

[4] M. Fiedler and V. Ptak, "On matrices with non-positive off-diagonal elements and positive principal minors," Czechoslovak Mathematics Journal 12 (1962) 382-400.

[5] D. Gale and H. Nikaido, "The Jacobian matrix and global univalence of mappings," Mathematische Annalen 159 (1965) 81-93.

[6] R. Howe and R. Stone, "Linear complementarity and the degree of mappings," in: B.C. Eaves, F.J. Gould, H.O. Peitgen and M.J. Todd, eds., Homotopy Methods and Global Convergence (Plenum, New York, 1983).

[7] L.M. Kelly and L.T. Watson, "Q-matrices and spherical geometry," Linear Algebra and its Applications 25 (1979) $175-190$.

[8] L.M. Kelly and L.T. Watson, "Erratum: Some perturbation theorems for Q-matrices," SIAM Journal of Applied Mathematics 34 (1978) 320-321.

[9] O.L. Mangasarian, Nonlinear Programming (McGraw-Hill, New York, 1969).

[10] W.D. Morris Jr. and J. Lawrence, "Geometric properties of hidden Minkowski matrices," SIAM Journal for Matrix Analysis and Applications 10 (1989) 229-232.

[11] K.G. Murty, "On the parametric complementarity problem," Engineering Summer Conference notes, University of Michigan (Ann Arbor, MI, 1971).

[12] K.G. Murty, "Computational complexity of complementary pivot methods," Mathematical Programming Study 7 (1978) 61-73.

[13] K.G. Murty, "On the linear complementarity problem," Methods of Operations Research 31 (1978) 425-439.

[14] K.G. Murty, Linear Complementarity, Linear and Nonlinear Programming (Heldermann, West Berlin, 1988).

[15] K.G. Murty, Linear Programming (Wiley, New York, 1983).

[16] K.G. Murty and Y. Fathi, "A critical index algorithm for nearest point problems in simplicial cones," Mathematical Programming 23 (1982) 206-215.

[17] J.S. Pang and R. Chandrasekaran, "Linear complementarity problems solvable by a polynomially bounded pivoting algorithm," Mathematical Programming Study 25 (1985) 13-27.

[18] L.T. Watson, "A variational approach to the linear complementarity problem," Doctoral dissertation, Department of Mathematics, University of Michigan (Ann Arbor, MI, 1974). 
[19] L.T. Watson, "Some perturbation theorems for Q-matrices," SIAM Journal of Applied Mathematics 31 (1976) 379-384.

[20] L.T. Watson, "An algorithm for the linear complementarity problem," International Journal of Computer Mathematics 6 (1978) 319-325.

[21] P. Wolfe, "Finding the nearest point in a polytope," Mathematical Programming 11 (1976) $128-149$. 